\title{
Covers for self-dual supercuspidal representations of the Siegel Levi subgroup of classical $p$-adic groups
}

\author{
David Goldberg; Philip Kutzko ${ }^{\S}$ and Shaun Stevens ${ }^{\dagger}$
}

\author{
9 August 2007
}

\begin{abstract}
We study components of the Bernstein decomposition of a $p$-adic classical group (with $p$ odd) with inertial support a self-dual positive level supercuspidal representation of a Siegel Levi subgroup. More precisely, we use the method of covers to construct a Bushnell-Kutzko type for such a component. A detailed knowledge of the Hecke algebra of the type should have number-theoretic implications.
\end{abstract}

\section{Introduction}

Let $F_{0}$ be a locally compact nonarchimedean local field, and $G$ the $F_{0}$-points of a connected reductive quasi-split algebraic group $\boldsymbol{G}$ defined over $F_{0}$. The smooth representation theory of $G$ plays a key role in the modern theory of automorphic forms, and is therefore of deep interest to number theorists. A theorem of Bernstein shows that, in the terminology of [7], it is enough to understand the subcategories $\mathfrak{R}^{\mathfrak{s}}(G)$ of smooth representations with supercuspidal support in a given inertial class $\mathfrak{s}$. The class $\mathfrak{s}=[L, \pi]_{G}$ is determined by a Levi subgroup $L \subset G$ and an irreducible supercuspidal representation $\pi$ of $L$. The theory of types and covers [7] gives one a method for trying to understand the subcategories $\mathfrak{R}^{\mathfrak{s}}(G)$. In particular, if one knows supercuspidal types for all Levi subgroups of $G$, and how to construct $G$-covers of all such types, then one has a complete set of types for $G$. Here, we study the case where $F_{0}$ has odd residual characteristic, $G$ is a classical group, and $\mathfrak{s}=[L, \pi]_{G}$ with $L$ a Siegel Levi subgroup and $\pi$ an irreducible supercuspidal representation fixed by the non-trivial Weyl group element. While this work gives a complete set of $G$-covers (and hence types) in this case, the theory cannot be considered complete without knowledge of the associated Hecke algebras. As shown in [16], a precise understanding of such Hecke algebras gives information on reducibility of induced representations and therefore determines poles of certain Langlands $L$-functions. These considerations will be the subject of future work.

Let $\boldsymbol{G}$ be any of the groups $\boldsymbol{S} \boldsymbol{p}_{2 M}, \boldsymbol{S} \boldsymbol{O}_{2 M}, \boldsymbol{S} \boldsymbol{O}_{2 M+1}, \boldsymbol{U}_{n}$ or $\boldsymbol{S} \boldsymbol{O}_{n}^{*}$, i.e., a symplectic, quasi-split special orthogonal group, or quasi-split unitary group. (Here $\boldsymbol{S O}_{n}^{*}$ stands for a quasi-split but nonsplit special orthogonal group.) Let $\boldsymbol{B}=\boldsymbol{T} \boldsymbol{N}$ be a Borel subgroup with unipotent radical $\boldsymbol{N}$ and

\footnotetext{
¥The first author was supported by NSF grant DMS0400958.

$\S$ The second author was supported by NSF grant DMS0101451.

${ }^{\dagger}$ The third author was supported by grant NAL/00779/G from the Nuffield Foundation.
} 
maximal torus $\boldsymbol{T}$. There is a choice of standard maximal parabolic subgroup $\boldsymbol{P}=\boldsymbol{L} \boldsymbol{U}$ containing $\boldsymbol{B}$ whose Levi component $\boldsymbol{L}$ is isomorphic to either $\boldsymbol{G} \boldsymbol{L}_{M} \times \boldsymbol{G}_{0}$, or $\boldsymbol{R e s}_{F / F_{0}}\left(\boldsymbol{G} \boldsymbol{L}_{M}\right) \times \boldsymbol{G}_{0}$; here $\boldsymbol{G}_{0}$ is an anisotropic group and $F / F_{0}$ is the quadratic extension defining the unitary group. We study the theory of types for the inertial class $\mathfrak{s}=[L, \pi]_{G}$, where $\pi$ is fixed by the non-trivial element $w_{0}$ of the Weyl group $N_{\boldsymbol{G}}(\boldsymbol{L}) / \boldsymbol{L}$. The work of Bushnell and Kutzko [6] gives one a type $\left(J_{L}, \lambda_{L}\right)$ for the inertial class $\mathfrak{s}_{L}=[L, \pi]_{L}$. The purpose of this paper is to construct a cover $(J, \lambda)$ of $\left(J_{L}, \lambda_{L}\right)$ for each group $\boldsymbol{G}$. (This generalizes the work of Blondel [3] when $\boldsymbol{G}=\boldsymbol{S} \boldsymbol{p}_{2 M}$, though her proofs are somewhat different.) Therefore, by [7, the pair $(J, \lambda)$ is a type for $\mathfrak{s}$. One then knows that the category $\mathfrak{R}^{\mathfrak{s}}(G)$ is isomorphic to the category $\mathcal{H}(G, \lambda)$-Mod of unital (left) modules over the $\lambda$-spherical Hecke algebra, and we also give the structure of this Hecke algebra.

This isomorphism of categories has implications both for the location of poles of certain $L$-functions, and for the classification of local galois representations. One example of this may be seen in recent work of Kutzko and Morris [16], where $\boldsymbol{G}$ is one of the groups $\boldsymbol{S} \boldsymbol{p}_{2 M}, \boldsymbol{S O}_{2 M}$ or $\boldsymbol{S O}_{2 M+1}$, and $\pi$ is a level zero self-dual supercuspidal representation. Specific information about the Hecke algebra $\mathcal{H}(G, \lambda)$ is employed to give a purely local proof of Shahidi's theorem on the reducibility of parabolic induction [21, which can also be described in terms of the poles of $L$-functions. On the other hand, recent work of Henniart [15, building on work of Harris and Taylor [12] and Henniart [14, relates this to the classification of local galois representations by their images (e.g. symplectic, orthogonal). In the case of the level zero representations in [16], the classification of galois representations obtained via the Hecke algebra isomorphism was known already. However, in the general situation of this paper, the corresponding classification of galois representations is not fully understood and has been sought for some time. We hope to address this problem in a sequel.

We now give a summary of the contents of the paper. In $\$ 1.1$, we review the theory of types for self-dual representations, via results of Blondel, which is crucial to the construction (see 3 , Proposition 2.2]). (Note that our use of the word self-dual is somewhat non-standard: it does not mean self-contragredient.) In $\$ 1.3$, we fix a self-dual representation $\pi$ of $L$, and use these results to define a well-adapted inner product on an $F$-vector space $V$, which defines the group $G$. In $\$ 1.4$, we show that there is a choice of simple stratum (see [6]), defining the type in $\pi$, which is particularly well suited. In particular, it gives rise to a skew semisimple stratum (see [24]) in $\operatorname{End}_{F}(V)$, which underpins the construction of $G$-cover.

We construct the $G$-cover $(J, \lambda)$ in $\$ 2$, following the recipe of [6, $\S 7]$. The first stages, when we are working with pro- $p$ groups, are performed first in $\operatorname{Aut}_{F}(V)$ (see \$2.1), and then transferred to $G$ using the Glauberman correspondence (see $\$ 2.2$ ). Indeed, the principle of the construction is the same as that of Blondel for symplectic groups [3], though the details are complicated by the presence of an anisotropic part. We diverge from [3] in the verification that $(J, \lambda)$ is indeed a $G$ cover, where we construct an invertible element $f$ in the Hecke algebra $\mathcal{H}(G, \lambda)$, which is supported on a strongly $(P, J)$-positive element in the centre of $L$ (see [7]). To do this, we use an argument which harks back to the work of Borel 4]: we find two invertible elements of the Hecke algebra $\mathcal{H}(G, \lambda)$, which each have support in a compact subgroup of $G$, and whose convolution is supported on a strongly positive element of $L$ (see Lemmas 2.10, 2.11); a suitable power of this is the required element $f$.

Finally, in 82.3 , we prove a result on the Hecke algebra $\mathcal{H}(G, \lambda)$, following the techniques and philosophy of [6, §5] (see also [19]). We show that the subalgebra of elements with support in a fixed maximal compact subgroup is isomorphic to an algebra of the form $\mathcal{H}\left(\mathcal{G}^{\prime}, \rho^{\prime}\right)$, where $\mathcal{G}^{\prime}$ is a (possibly disconnected) classical group over a finite field, and $\rho^{\prime}$ is an irreducible cuspidal 
representation of the Siegel Levi subgroup of $\mathcal{G}^{\prime}$. In many cases, the calculations in [16] will give the parameters of these Hecke algebras; in general, the calculations will be similar. This will simplify the computation of the structure of the Hecke algebra $\mathcal{H}(G, \lambda)$; these specific calculations, and their implications to the classification of galois representations, are left to future work.

Acknowledgements We would like to thank Kazutoshi Kariyama, for pointing out some mistakes, and, especially, the anonymous referees, whose careful reading, apposite comments and numerous corrections and suggestions have led to a much clearer paper.

\section{Preliminaries}

Let $F$ be a locally compact nonarchimedean local field. Let $\mu$ be an automorphism of $F$ with $\mu^{2}=1$; we allow the possibility that $\mu$ is trivial. We set $F_{0}=F^{\mu}$ to be the fixed points of $\mu$. Let $\mathfrak{o}_{F}$ be the ring of integers in $F$, and $\mathfrak{p}_{F}$ the maximal ideal in $\mathfrak{o}_{F}$. Denote by $k_{F}$ the residual field $\mathfrak{o}_{F} / \mathfrak{p}_{F}$, and let $q_{F}=\left|k_{F}\right|$. We adopt similar notation for $F_{0}$ and for any extension of $F_{0}$.

Let $p$ denote the characteristic of $k_{F}$. We assume that $p$ is not 2 throughout.

For $r$ a real number, we write $\lfloor r\rfloor$ for the greatest integer less than or equal to $r$, and $\lceil r\rceil$ for the least integer greater than or equal to $r$.

\subsection{Self-dual representations of $\mathrm{GL}_{\mathrm{M}}(\mathrm{F})$}

We begin by looking at the self-dual representations of $G L_{M}(F)$, following Blondel [3, §2.2]. Let $W$ be an $M$-dimensional $F$-vector space with basis $\mathcal{B}=\left\{\mathbf{w}_{1}, \ldots, \mathbf{w}_{M}\right\}$, equipped with the nondegenerate hermitian form $\langle,\rangle_{W}$ given by $\left\langle\mathbf{w}_{i}, \mathbf{w}_{j}\right\rangle_{W}=\delta_{i+j, M+1}$. Denote by $\widetilde{\varepsilon}_{W}$ the induced adjoint involution on $A_{W}=\operatorname{End}_{F}(W)$ and by $\varepsilon_{W}$ the involution $g \mapsto \widetilde{\varepsilon}_{W}\left(g^{-1}\right)$ of $G_{W}=\operatorname{Aut}_{F}(W) \cong$ $G L_{M}(F)$. Then, writing $a \in A_{W}$ with respect to the basis $\mathcal{B}$, we have

$$
\widetilde{\varepsilon}_{W}(a)={ }^{\dagger} a^{\mu},
$$

where $a^{\mu} \in A_{W}$ is obtained by applying $\mu$ to the coefficients of $a$, and ${ }^{\dagger}$ denotes transpose with respect to the off-diagonal. For $\rho$ a representation of a subgroup $J$ of $G_{W}$, we write $\rho^{\varepsilon_{W}}$ for the representation of $\varepsilon_{W}(J)$ given by $\rho^{\varepsilon_{W}}\left(\varepsilon_{W}(j)\right)=\rho(j)$, for $j \in J$.

Let $\pi$ be an irreducible supercuspidal representation of $G_{W}$ such that $\pi \simeq \pi^{\varepsilon_{W}}$. Note that, if $F=F_{0}$, then, by Gel'fand and Kazhdan [11, this is equivalent to $\pi$ being self-contragredient. Let $\left(J_{W}, \lambda_{W}\right)$ be a maximal simple type in $G_{W}$ corresponding to the inertial equivalence class $\left[G_{W}, \pi\right]_{G_{W}}$. We will need to use the construction of $\lambda_{W}$ quite explicitly so we recall it briefly here (see [6] for more details).

It begins with a simple stratum $\left[\mathfrak{A}_{W}, n_{W}, 0, \beta\right]$, where $\mathfrak{A}_{W}$ is a principal hereditary $\mathfrak{o}_{F}$-order in $A_{W}$, with Jacobson radical $\mathfrak{P}_{W}$, and $n_{W} \in \mathbb{N}$ is such that $\beta \in \mathfrak{P}_{W}^{-n_{W}} \backslash \mathfrak{P}_{W}^{1-n_{W}}$. Further, $E=F[\beta]$ is a field extension of $F$ and $E^{\times}$normalizes $\mathfrak{A}_{W}$. We set $B_{W}$ to be the $A_{W}$-centralizer of $E$ and put $\mathfrak{B}_{W}=\mathfrak{A}_{W} \cap B_{W}$, a maximal hereditary $\mathfrak{o}_{E}$-order in $B_{W}$. We also fix a uniformizer $\varpi_{E}$ of $E$.

From the stratum are defined certain subgroups $H_{W}^{k}=H^{k}\left(\beta, \mathfrak{A}_{W}\right)$ and $J_{W}^{k}=J^{k}\left(\beta, \mathfrak{A}_{W}\right)$, for $k \geq 0$, along with some sets $\mathcal{C}\left(\mathfrak{A}_{W}, k, \beta\right)$ of characters of $H_{W}^{k+1}$ called simple characters (see [6, §3]). The construction of the type continues with a simple character $\theta_{W} \in \mathcal{C}\left(\mathfrak{A}_{W}, 0, \beta\right)$. There is then a unique irreducible representation $\eta_{W}$ of $J_{W}^{1}$ which contains $\theta_{W}$. 
Now we take $\kappa_{W}$ to be a $\beta$-extension of $\eta_{W}$, that is, one of a certain family of representations of $J_{W}=J_{W}^{0}$ which restrict to $\eta_{W}$. We also recall the construction of $\kappa_{W}$ here, from [6, §5.1-2]. Let $\mathfrak{B}_{W}^{\mathrm{m}}$ be a minimal hereditary $\mathfrak{o}_{E}$-order in $B_{W}$ contained in $\mathfrak{B}_{W}$ and let $\mathfrak{A}_{W}^{\mathrm{m}} \subset \mathfrak{A}_{W}$ be the unique hereditary $\mathfrak{o}_{F}$-order in $A_{W}$ which is normalized by $E^{\times}$and such that $\mathfrak{A}_{W}^{\mathfrak{m}} \cap B_{W}=\mathfrak{B}_{W}^{\mathrm{m}}$. Then $\left[\mathfrak{A}_{W}^{\mathrm{m}}, n_{W}^{\mathrm{m}}, 0, \beta\right]$ is also a simple stratum, for some integer $n_{W}^{\mathrm{m}}$, and we can define simple characters associated to this stratum also. Moreover, there is a canonical bijection $\tau_{\mathfrak{A}_{W}, \mathfrak{A}_{W}^{m}, \beta}: \mathcal{C}\left(\mathfrak{A}_{W}, 0, \beta\right) \rightarrow$ $\mathcal{C}\left(\mathfrak{A}_{W}^{\mathrm{m}}, 0, \beta\right)$ (see [6, §3.6]). Let $\theta_{W}^{\mathrm{m}}$ be the transfer of $\theta_{W}$ under this bijection. There is a unique irreducible representation $\eta_{W}^{\mathrm{m}}$ of $J^{1}\left(\beta, \mathfrak{A}_{W}^{\mathrm{m}}\right)$ which contains $\theta_{W}^{\mathrm{m}}$.

Now we form the group $\widetilde{J}_{W}^{1}=U^{1}\left(\mathfrak{B}_{W}^{\mathrm{m}}\right) J_{W}^{1}$. There is a unique representation $\widetilde{\eta}_{W}$ of $\widetilde{J}_{W}^{1}$ such that $\left.\widetilde{\eta}_{W}\right|_{J_{W}^{1}}=\eta_{W}$ and $\widetilde{\eta}_{W}, \eta_{W}^{\mathrm{m}}$ induce equivalent irreducible representations of $U^{1}\left(\mathfrak{A}_{W}^{\mathrm{m}}\right)$. Then $\kappa_{W}$ is any representation of $J_{W}$ such that $\left.\kappa_{W}\right|_{\widetilde{J}_{W}^{1}}=\widetilde{\eta}_{W}$. Finally, $J_{W} / J_{W}^{1} \cong U\left(\mathfrak{B}_{W}\right) / U^{1}\left(\mathfrak{B}_{W}\right)$ is isomorphic to $G L_{r}\left(k_{E}\right)$, where $r=M /[E: F]$. Then there is a cuspidal representation $\rho_{W}$ of $J_{W} / J_{W}^{1}$ such that

$$
\lambda_{W}=\kappa_{W} \otimes \rho_{W}
$$

By conjugating $\left(J_{W}, \lambda_{W}\right)$ if necessary, we may and do assume $\mathfrak{A}_{W}$ is standard; that is, with respect to our chosen basis $\mathcal{B}$, it consists of matrices with entries in $\mathfrak{o}_{F}$ which are upper block triangular modulo $\mathfrak{p}_{F}$. Note that this means that $\widetilde{\varepsilon}_{W}\left(\mathfrak{A}_{W}\right)=\mathfrak{A}_{W}$.

Proposition 1.1 (cf. [3, 2.2 Proposition]). (i) There exists $\sigma \in U\left(\mathfrak{A}_{W}\right)$ such that $J_{W}$ is stable under $\widetilde{\sigma}: g \rightarrow \sigma \varepsilon_{W}(g) \sigma^{-1}$ and $\lambda_{W}$ is equivalent to $\lambda_{W} \circ \widetilde{\sigma}$.

(ii) Such an element $\sigma$ is unique up to left multiplication by $J_{W}$. It satisfies:

(a) $\sigma \varepsilon_{W}(\sigma) \in J_{W}$ and $\varpi_{E}^{-1} \sigma \varepsilon_{W}\left(\varpi_{E}^{-1} \sigma\right) \in J_{W}$.

(b) The map $\widetilde{\sigma}$ stabilizes $H_{W}^{1}$ and $J_{W}^{1}$ and we have $\theta_{W}=\theta_{W} \circ \widetilde{\sigma}$.

(c) The lattices $\mathfrak{J}_{W}, \mathfrak{H}_{W}^{1}$ are stable under $X \mapsto \sigma \widetilde{\varepsilon}_{W}(X) \sigma^{-1}$.

The proof is identical to that of [3, 2.2 Proposition]. Note that we will actually have to be somewhat more careful in our choices of $\beta$ and $\sigma$, as in [3, 2.3 Corollary] - see Remark 1.4 for more details.

\subsection{Simple characters}

We record here the following useful lemma from [3, 4.3 Lemma]. Note that this uses very strongly the condition that $p \neq 2$.

Lemma 1.2 ([3, 4.3 Lemma 1]). Suppose $V^{\prime \prime}$ is any F-vector space and $\left[\mathfrak{A}^{\prime \prime}, n^{\prime \prime}, 0, \beta^{\prime \prime}\right]$ is a simple stratum in $A^{\prime \prime}=\operatorname{End}_{F}\left(V^{\prime \prime}\right)$, where $\mathfrak{A}^{\prime \prime}$ is a hereditary $\mathfrak{o}_{F}$-order in $V^{\prime \prime}$.

(i) $\left[\mathfrak{A}^{\prime \prime}, n^{\prime \prime}, 0, \frac{1}{2} \beta^{\prime \prime}\right]$ is a simple stratum in $A^{\prime \prime}$, with $H^{k}\left(\frac{1}{2} \beta^{\prime \prime}, \mathfrak{A}^{\prime \prime}\right)=H^{k}\left(\beta^{\prime \prime}, \mathfrak{A}^{\prime \prime}\right)$, for each $k \geq 0$, and similarly for $J^{k}$.

(ii) For each $m \geq 0$, the map $\theta \mapsto \theta^{2}$ is a bijection from $\mathcal{C}\left(\mathfrak{A}^{\prime \prime}, m, \frac{1}{2} \beta^{\prime \prime}\right)$ onto $\mathcal{C}\left(\mathfrak{A}^{\prime \prime}, m, \beta^{\prime \prime}\right)$ which is compatible with the canonical bijections $\tau$ of [6, §3.6]. We denote the inverse bijection by $\theta \mapsto \theta^{1 / 2}$. 
We will write $\theta_{L}=\theta_{W}^{1 / 2} \in \mathcal{C}\left(\mathfrak{A}_{W}, 0, \frac{1}{2} \beta\right)$ and $\theta_{L}^{\mathrm{m}}=\left(\theta_{W}^{\mathrm{m}}\right)^{1 / 2} \in \mathcal{C}\left(\mathfrak{A}_{W}^{\mathrm{m}}, 0, \frac{1}{2} \beta\right)$. We also let $\eta_{L}$ be the unique irreducible representation of $J^{1}\left(\beta, \mathfrak{A}_{W}\right)$ which contains $\theta_{L}$, and likewise $\eta_{L}^{\mathrm{m}}$.

Note also that we have $\left(\theta_{L} \circ \widetilde{\sigma}\right)^{2}=\theta_{L}^{2} \circ \widetilde{\sigma}=\theta_{W}$. In particular, since the squaring map is a bijection, we see that $\theta_{L} \circ \widetilde{\sigma}=\theta_{L}$.

\subsection{Classical groups}

Let $V_{0}$ be an $F$-vector space equipped with a nondegenerate anisotropic $\nu$-hermitian form $\langle,\rangle_{0}$, with $\nu= \pm 1$. Thus $\left\langle\mathbf{v}_{0}, \mathbf{w}_{0}\right\rangle_{0}=\nu\left\langle\mathbf{w}_{0}, \mathbf{v}_{0}\right\rangle_{0}^{\mu}$, for all $\mathbf{v}_{0}, \mathbf{w}_{0} \in V_{0}$. We write $G_{0}=\operatorname{Aut}_{F}\left(V_{0}\right)$ and denote by $\bar{G}_{0}^{+}$the (anisotropic) group corresponding to the form $\langle,\rangle_{0}$ :

$$
\bar{G}_{0}^{+}:=\left\{g_{0} \in G_{0}:\left\langle g_{0} \mathbf{v}_{0}, g_{0} \mathbf{w}_{0}\right\rangle_{0}=\left\langle\mathbf{v}_{0}, \mathbf{w}_{0}\right\rangle_{0} \text { for all } \mathbf{v}_{0}, \mathbf{w}_{0} \in V_{0}\right\} .
$$

We put $\bar{G}_{0}:=\left\{g_{0} \in \bar{G}_{0}^{+}: N_{F / F_{0}} \circ \operatorname{det}_{G_{0} / F}\left(g_{0}\right)=1\right\}$. We allow the possibility that $V_{0}=\{0\}$.

Set $V=W \oplus V_{0} \oplus W$ and define a form $\langle$,$\rangle on V$ by

$$
\left\langle\left(\mathbf{v}_{1}, \mathbf{v}_{0}, \mathbf{v}_{2}\right),\left(\mathbf{w}_{1}, \mathbf{w}_{0}, \mathbf{w}_{2}\right)\right\rangle=\left\langle\mathbf{v}_{1}, \sigma^{-1} \mathbf{w}_{2}\right\rangle_{W}+\left\langle\mathbf{v}_{0}, \mathbf{w}_{0}\right\rangle_{0}+\nu\left\langle\sigma^{-1} \mathbf{v}_{2}, \mathbf{w}_{1}\right\rangle_{W},
$$

for $\mathbf{v}_{1}, \mathbf{v}_{2}, \mathbf{w}_{1}, \mathbf{w}_{2} \in W$ and $\mathbf{v}_{0}, \mathbf{w}_{0} \in V_{0}$, where $\sigma$ is the element given by Proposition 1.1. Note that $\langle$,$\rangle is now a nondegenerate \nu$-hermitian form in which the two copies of $W$ are (dual) maximal isotropic subspaces, and $V_{0}$ is the maximal anisotropic subspace. Let $N=\operatorname{dim}_{F} V$; then $N=$ $2 M+D$, where $D=\operatorname{dim}_{F} V_{0}$.

We put $A=\operatorname{End}_{F}(V) \cong \mathbb{M}(N, F)$. We set $G=A^{\times}=\operatorname{Aut}_{F}(V) \cong G L_{N}(F)$ and

$$
\bar{G}^{+}=\{g \in G:\langle g \mathbf{v}, g \mathbf{w}\rangle=\langle\mathbf{v}, \mathbf{w}\rangle \text { for all } \mathbf{v}, \mathbf{w} \in V\},
$$

a unitary, symplectic or orthogonal group over $F_{0}$. We also put

$$
\bar{G}:=\left\{g \in \bar{G}^{+}: N_{F / F_{0}} \circ \operatorname{det}_{G / F}(g)=1\right\} .
$$

More generally, for $H$ a subgroup of $G$, we will write $\bar{H}^{+}$for the intersection $\bar{H}^{+}=H \cap \bar{G}^{+}$, and $\bar{H}$ for the intersection $\bar{H}=H \cap \bar{G}$.

We denote by $\widetilde{\varepsilon}$ the adjoint involution on $A$ determined by the form; that is, for $a \in A, \widetilde{\varepsilon}(a)$ is the unique element of $A$ such that

$$
\langle a v, w\rangle=\langle v, \widetilde{\varepsilon}(a) w\rangle, \quad \text { for all } v, w, \in V,
$$

We have an involution $\varepsilon$ on $G$ given by $\varepsilon(g)=\widetilde{\varepsilon}\left(g^{-1}\right)$, for $g \in G$, so that $\bar{G}^{+}=G^{\varepsilon}$.

Denote by $\phi_{1}$ (respectively $\phi_{2}$ ) the embedding of $A_{W}$ into $A$ via the embedding of $W$ into the first (respectively last) factor of $V=W \oplus V_{0} \oplus W$, so that $\phi_{1}(a)\left(\mathbf{v}_{1}, \mathbf{v}_{0}, \mathbf{v}_{2}\right)=\left(a \mathbf{v}_{1}, \mathbf{v}_{0}, \mathbf{v}_{2}\right)$. The involution $\widetilde{\varepsilon}$ interchanges the images of these two embeddings, and a straightforward calculation shows that $\widetilde{\varepsilon}\left(\phi_{1}(a)\right)=\phi_{2}\left(\sigma \varepsilon_{W}(a) \sigma^{-1}\right)=\phi_{2}(\widetilde{\sigma}(a))$, using the notation of Proposition 1.1 .

Given a representation $\rho$ of a subgroup $J$ of $G$, we denote by $\rho^{\varepsilon}$ the representation of $\varepsilon(J)$ given by $\rho^{\varepsilon}(\varepsilon(j))=\rho(j)$, for $j \in J$.

For $L$ an $\mathfrak{o}_{F}$-lattice in $V$, we define its dual lattice to be

$$
L^{\#}=\left\{v \in V:\langle v, L\rangle \subset \mathfrak{p}_{F}\right\} .
$$


We note that, since $\bar{G}_{0}^{+}$stabilizes lattices $L_{0} \supset L_{0}^{\#} \supset \mathfrak{p}_{F} L_{0}$ in $V_{0}$ (see [17, §1.8]), it is contained in a maximal $\varepsilon$-stable compact open subgroup of $G_{0}$ (namely, the $G_{0}$-stabilizer of these lattices).

Let $P$ be the parabolic subgroup of $G$ stabilizing the self-dual flag

$$
\{0\} \subsetneq W \oplus\{0\} \oplus\{0\} \subseteq(W \oplus\{0\} \oplus\{0\})^{\perp}=W \oplus V_{0} \oplus\{0\} \subsetneq V,
$$

with unipotent radical $U$. Let $L$ denote the Levi component of $P$ which stabilizes each copy of $W$ along with $V_{0}$. So $L \cong G L_{M}(F) \times G L_{D}(F) \times G L_{M}(F)$. Let $P_{-}$denote the opposite parabolic subgroup, $P_{-}=L U_{-}$. We also put $A_{L}=A_{W} \oplus A_{0} \oplus A_{W}=\operatorname{Lie}(L)$.

We set $\bar{P}=P \cap \bar{G}$, the Siegel parabolic subgroup of $\bar{G}$, with unipotent radical $\bar{U}=U \cap \bar{G}$, and $\bar{L}=L \cap \bar{G}$, a Levi component of $\bar{P}$. Then $\bar{L} \cong G L_{M}(F) \times \bar{G}_{0}$; in block matrix form, we identify $G_{W} \times \bar{G}_{0}$ with $\bar{L}$ via the isomorphism

$$
i\left(g, g_{0}\right)=\left(\begin{array}{ccc}
g & 0 & 0 \\
0 & g_{0} & 0 \\
0 & 0 & \widetilde{\sigma}(g)
\end{array}\right), \quad \text { for } g \in G_{W}, g_{0} \in \bar{G}_{0}
$$

Note here that we are writing matrices with respect to the non-Witt basis $\mathcal{B} \cup \mathcal{B}_{0} \cup \mathcal{B}$ of $V=$ $W \oplus V_{0} \oplus W$, for $\mathcal{B}_{0}$ any basis of $V_{0}$. We choose to write all matrices with respect to this basis, rather than with respect to the Witt basis $\mathcal{B} \cup \mathcal{B}_{0} \cup \sigma \mathcal{B}$.

If $\rho$ is a representation of a subgroup $J_{W}$ of $G_{W}$, and $\bar{J}_{0}$ is a subgroup of $\bar{G}_{0}$, then we denote by $i(\rho)$ the representation of $i\left(J_{W} \times \bar{J}_{0}\right)$ given by $i(\rho)\left(i\left(j, j_{0}\right)\right)=\rho(j)$, for $j \in J_{W}, j_{0} \in \bar{J}_{0}$. If the group $\bar{J}_{0}$ is not specified then we take it to be the whole of $\bar{G}_{0}$.

More generally, if $\bar{J}$ is a subgroup of $\bar{G}$ such that $\bar{J} \cap \bar{L}=i\left(J_{W} \times \bar{J}_{0}\right)$ and $\bar{J}$ has an Iwahori decomposition with respect to $(\bar{L}, \bar{P})$, then we denote by $\widetilde{i(\rho)}$ the representation of $\bar{J}$ given by

$$
\widetilde{i(\rho)}\left(u_{-} l u\right)=i(\rho)(l), \quad \text { for } u_{-} \in \bar{J} \cap \bar{U}_{-}, l \in \bar{J} \cap \bar{L}, u \in \bar{J} \cap \bar{U},
$$

whenever this defines a representation.

It will also be useful, later, to put $V^{\prime}=W \oplus\{0\} \oplus W \subset V$, equipped with the restriction of the form $\langle$,$\rangle . We put A^{\prime}=\operatorname{End}_{F}\left(V^{\prime}\right), G^{\prime}=\operatorname{Aut}_{F}\left(V^{\prime}\right), \varepsilon$ the involution of $G^{\prime}$ associated to the form, and $\bar{G}^{\prime}=\left(G^{\prime}\right)^{\varepsilon}$. We also let $P^{\prime}$ be the maximal parabolic subgroup of $G$ which stabilizes the flag

$$
\{0\} \subsetneq V^{\prime} \subsetneq V
$$

with unipotent radical $U^{\prime}$. Let $L^{\prime}$ denote the Levi component of $P^{\prime}$ which stabilizes the decomposition $V=V^{\prime} \oplus V_{0}$, so that $L^{\prime} \cong G^{\prime} \times G_{0}$, and let $P^{\prime-}$ denote the opposite parabolic subgroup, with unipotent radical $U^{\prime-}$. We note that, while $L^{\prime}$ is stable under the involution $\varepsilon, \bar{L}^{\prime}$ is not a Levi subgroup of $\bar{G}$.

We consider the inertial class $\mathfrak{s}_{\bar{L}}=[\bar{L}, i(\pi)]_{\bar{L}}$ and a type $\left(J_{\bar{L}}, \lambda_{\bar{L}}\right)$ for it, where

$$
J_{\bar{L}}:=i\left(J_{W}\right)=i\left(J_{W} \times \bar{G}_{0}\right) \quad \text { and } \quad \lambda_{\bar{L}}:=i\left(\lambda_{W}\right) .
$$

We are going to construct a $\bar{G}$-cover of this type, which will give a $\bar{G}$-type for the inertial class $\mathfrak{s}=[\bar{L}, i(\pi)]_{\bar{G}}$. 


\subsection{Semisimple characters}

We continue with the notation of the previous sections. Let $\mathcal{L}_{W}=\left\{L_{k}^{W}: k \in \mathbb{Z}\right\}$ be the $\mathfrak{o}_{E}$-lattice chain in $W$ corresponding to $\mathfrak{A}_{W}$, so that

$$
\mathfrak{A}_{W}=\left\{a \in A_{W}: a L_{k}^{W} \subset L_{k}^{W} \text { for all } k \in \mathbb{Z}\right\},
$$

normalized so that $L_{0}^{W}=\mathfrak{o}_{F} \mathbf{w}_{1} \oplus \cdots \oplus \mathfrak{o}_{F} \mathbf{w}_{M}$. Let $e_{W}$ denote the $\mathfrak{o}_{F}$-period of $\mathfrak{A}_{W}$. We define $\mathcal{L}^{\prime}$ to be the $\mathfrak{o}_{E}$-lattice chain of $\mathfrak{o}_{F}$-period $2 e_{W}$ in $V^{\prime}$ given by

$$
\cdots \supset L_{k}^{W} \oplus L_{k}^{W} \supset L_{k}^{W} \oplus L_{k+1}^{W} \supset L_{k+1}^{W} \oplus L_{k+1}^{W} \supset \cdots
$$

It is straightforward to check, since $\sigma \in U\left(\mathfrak{A}_{W}\right)$, we have

$$
\left(L_{k}^{W} \oplus L_{k}^{W}\right)^{\#}=L_{e_{W}-k}^{W} \oplus L_{e_{W}-k}^{W} \quad \text { and } \quad\left(L_{k}^{W} \oplus L_{k+1}^{W}\right)^{\#}=L_{e_{W}-k-1}^{W} \oplus L_{e_{W}-k}^{W}
$$

so that $\mathcal{L}^{\prime}$ is a self-dual lattice chain. We write $\mathcal{L}^{\prime}=\left\{L_{k}^{\prime}: k \in \mathbb{Z}\right\}$, where we number the lattices so that $L_{0}^{\prime}=L_{\left\lfloor\frac{e_{W}}{2}\right\rfloor}^{W} \oplus L_{\left\lceil\frac{{ }^{e} W}{2}\right\rceil}^{W}=\left(L_{0}^{\prime}\right)^{\#}$. Let $\mathfrak{A}^{\prime}=\mathfrak{a}_{0}\left(\mathcal{L}^{\prime}\right)$ be the $\mathfrak{o}_{F}$-order associated with $\mathcal{L}^{\prime}$.

Now let $\Lambda^{\prime}$ be the $\mathfrak{o}_{E}$-lattice sequence of period $4 e_{W}$ in $V^{\prime}=W \oplus W$ given by

$$
\Lambda^{\prime}(k)=L_{\left\lfloor\frac{k}{2}\right\rfloor}^{\prime}
$$

so that every lattice of $\mathcal{L}^{\prime}$ occurs twice in the sequence and $\Lambda^{\prime}(k)^{\#}=\Lambda^{\prime}(1-k)$, for $k \in \mathbb{Z}$.

We consider the element $\frac{1}{2} \beta \oplus \frac{1}{2} \beta \in A_{W} \oplus A_{W}$, which we will also call $\frac{1}{2} \beta$. Then $\left[\mathcal{L}^{\prime}, 2 n_{W}, 0, \frac{1}{2} \beta\right]$ and $\left[\Lambda^{\prime}, 4 n_{W}, 0, \frac{1}{2} \beta\right]$ are both simple strata in $A^{\prime}$ so we can define the orders $\mathfrak{H}, \mathfrak{J}$ and the groups $H^{k}, J^{k}$ for them, and also simple characters (see [8]). These are in fact the same, up to a scaling of the index (loc. cit.); so, for example,

$$
H^{k}\left(\frac{1}{2} \beta, \Lambda^{\prime}\right)=H^{\left\lceil\frac{k}{2}\right\rceil}\left(\frac{1}{2} \beta, \mathcal{L}^{\prime}\right) .
$$

In particular, $H^{1}\left(\frac{1}{2} \beta, \Lambda^{\prime}\right)=H^{1}\left(\frac{1}{2} \beta, \mathcal{L}^{\prime}\right)$ and we shall denote this group $H^{\prime 1}$. Similarly, we put $J^{\prime 1}=J^{1}\left(\frac{1}{2} \beta, \Lambda^{\prime}\right)=J^{1}\left(\frac{1}{2} \beta, \mathcal{L}^{\prime}\right)$ and $J^{\prime}=J\left(\frac{1}{2} \beta, \Lambda^{\prime}\right)=J\left(\frac{1}{2} \beta, \mathcal{L}^{\prime}\right)$. Likewise, the simple characters of $H^{\prime 1}$ are the same: $\mathcal{C}\left(\Lambda^{\prime}, 0, \frac{1}{2} \beta\right)=\mathcal{C}\left(\mathcal{L}^{\prime}, 0, \frac{1}{2} \beta\right)$.

Moreover, the groups $H^{\prime 1}, J^{\prime 1}$ and $J^{\prime}$ are described in [3, 2.2 Lemma]. So, for example, with respect to the basis $\mathcal{B} \cup \mathcal{B}$ of $V^{\prime}$ (which, we recall, is not a Witt basis),

$$
H^{\prime 1}=\left(\begin{array}{cc}
H_{W}^{1} & \mathfrak{J}_{W} \\
\varpi_{E} \mathfrak{J}_{W} & H_{W}^{1}
\end{array}\right) \quad \text { and } \quad J^{\prime 1}=\left(\begin{array}{cc}
J_{W}^{1} & \varpi_{E}^{-1} \mathfrak{H}_{W} \\
\mathfrak{H}_{W} & J_{W}^{1}
\end{array}\right)
$$

Finally, we have the bijection $\tau_{\mathfrak{A}_{W}, \mathfrak{A}^{\prime}, \frac{1}{2} \beta}: \mathcal{C}\left(\mathfrak{A}_{W}, 0, \frac{1}{2} \beta\right) \rightarrow \mathcal{C}\left(\mathfrak{A}^{\prime}, 0, \frac{1}{2} \beta\right)$ from [6, §3.6]. Let $\theta^{\prime}$ be the image of $\theta_{L}$ under this map. Note that, by [6, $\left.\S 7.1-2\right], \theta^{\prime}$ is trivial on $H^{\prime 1} \cap U$ and $H^{\prime 1} \cap U_{-}$, while

$$
\left.\theta^{\prime}\right|_{H^{\prime 1} \cap L}=\theta_{L} \otimes \theta_{L} .
$$

It is straightforward to check that Proposition 1.1. together with our definition of the form $\langle$,$\rangle ,$ implies that $\theta^{\prime}$ is fixed by the involution $\varepsilon$ (cf. [3, 2.3 Corollary]).

Now let $\mathcal{L}_{0}$ be the unique self-dual $\mathfrak{o}_{F}$-lattice chain in $V_{0}$

$$
\cdots \supseteq L_{0} \supseteq L_{0}^{\#} \supseteq \mathfrak{p}_{F} L_{0} \supseteq \mathfrak{p}_{F} L_{0}^{\#} \supseteq \cdots .
$$


Note that we may have $L_{0}=L_{0}^{\#}$ or $L_{0}^{\#}=\mathfrak{p}_{F} L_{0}$ so this lattice chain has $\mathfrak{o}_{F}$-period $e_{0}=1$ or 2 . Let $\Lambda_{0}$ be the self-dual $\mathfrak{o}_{F}$-lattice sequence of period $4 e_{W}$ given by

$$
\Lambda_{0}(k)= \begin{cases}\mathfrak{p}_{F}^{j} L_{0} & \text { if }\left\lceil\frac{k}{2 e_{W}}\right\rceil=2 j \\ \mathfrak{p}_{F}^{j} L_{0}^{\#} & \text { if }\left\lceil\frac{k}{2 e_{W}}\right\rceil=2 j+1,\end{cases}
$$

so that every lattice of $\mathcal{L}_{0}$ appears with equal multiplicity $4 e_{W} / e_{0}$. Note also that $\Lambda_{0}(k)^{\#}=$ $\Lambda_{0}(1-k)$, for $k \in \mathbb{Z}$. Moreover, the filtration of $A_{0}$ determined by $\Lambda_{0}$ is the same as that determined by $\mathcal{L}_{0}$ up to a scaling of the index. In particular, $\mathfrak{a}_{0}\left(\Lambda_{0}\right)=\mathfrak{A}\left(\mathcal{L}_{0}\right)$ and $\mathfrak{a}_{1}\left(\Lambda_{0}\right)=\mathfrak{P}\left(\mathcal{L}_{0}\right)$.

Finally, we define $\Lambda$ to be the $\mathfrak{o}_{F}$-lattice sequence in $V$ given by

$$
\Lambda(k)=\Lambda^{\prime}(k) \oplus \Lambda_{0}(k), \quad \text { for } k \in \mathbb{Z} .
$$

Then, by construction, $\Lambda$ is self-dual and of $\mathfrak{o}_{F}$-period $e=4 e_{W}$. We consider the element $\frac{1}{2} \beta \oplus 0 \in$ $A^{\prime} \oplus A_{0}$ (in fact, in $A_{L}$ ); by abuse of notation, we will still call this element $\frac{1}{2} \beta$. Then $\left[\Lambda, n, 0, \frac{1}{2} \beta\right]$ is a semisimple stratum in $A$, where $n=4 n_{W}$. (See [24, §3.1] for the definition of semisimple stratum, which is more general than the definition in [22, §3.3]; in particular, null strata are thought of as simple strata so, alternatively, the definition in [22] could be used with "simple" replaced by "simple or null" everywhere. The results of [22] all remain valid in this situation - the proofs are the same and they are also proved in [24].)

We put $J=J\left(\frac{1}{2} \beta, \Lambda\right)$ (see [22] or [24]) and similarly for $J^{1}, H^{1}, \mathfrak{J}, \mathfrak{H}$ etc. In matrix form we have

$$
\mathfrak{J}=\left(\begin{array}{ccc}
\mathfrak{J}_{W} & \mathfrak{a}_{\lceil n / 2\rceil}(\Lambda) & \varpi_{E}^{-1} \mathfrak{H}_{W}^{1} \\
\mathfrak{a}_{\lceil n / 2\rceil}(\Lambda) & \mathfrak{A}\left(\mathcal{L}_{0}\right) & \mathfrak{a}_{\lceil n / 2\rceil}(\Lambda) \\
\mathfrak{H}_{W}^{1} & \mathfrak{a}_{\lceil n / 2\rceil}(\Lambda) & \mathfrak{J}_{W}
\end{array}\right) \quad \text { and } \quad \mathfrak{H}=\left(\begin{array}{ccc}
\mathfrak{H}_{W} & \mathfrak{a}_{\lfloor n / 2\rfloor}(\Lambda) & \mathfrak{J}_{W}^{1} \\
\mathfrak{a}_{\lfloor n / 2\rfloor}(\Lambda) & \mathfrak{A}\left(\mathcal{L}_{0}\right) & \mathfrak{a}_{\lfloor n / 2\rfloor}(\Lambda) \\
\varpi_{E} \mathfrak{J}_{W}^{1} & \mathfrak{a}_{\lfloor n / 2\rfloor}(\Lambda) & \mathfrak{H}_{W}
\end{array}\right)
$$

and there are similar decompositions for $\mathfrak{J}^{k}$ and $\mathfrak{H}^{k}, k \geq 1$. Since $J^{\prime}$ is stable under the involution $\varepsilon$ and $\Lambda$ is self-dual, we see that $J$ is stable under the involution $\varepsilon$, and likewise for $J^{1}$ and $H^{1}$.

Let $\theta$ be the unique semisimple character (see [22, §3.3] or [24, §3.2]) of $H^{1}$ such that

$$
\left.\theta\right|_{H^{\prime 1}}=\theta^{\prime} \text {. }
$$

Now $\theta$ is trivial on $H^{1} \cap U^{\prime}$ and $H^{1} \cap{U^{\prime}}^{-}$by definition so, since $\theta^{\prime}$ is trivial on $H^{\prime 1} \cap U$ and $H^{\prime 1} \cap U_{-}$, we see that $\theta$ is in fact trivial on $H^{1} \cap U$ and $H^{1} \cap U_{-}$. Since $\left.\theta\right|_{H^{\prime 1}}$ is fixed by $\varepsilon$ and $\left.\theta\right|_{U^{1}\left(\mathcal{L}_{0}\right)}$ is trivial (by definition), we see that $\theta$ is fixed by $\varepsilon$. Moreover, since $\theta_{L}^{2}=\theta_{W}$, we have

$$
\left.\theta\right|_{\bar{H}^{1}}=\widetilde{i\left(\theta_{W}\right)}
$$

Proposition 1.3 (cf. [3, 2.3 Theorem]). There exists a semisimple stratum $[\Lambda, n, 0, \alpha]$ in $A$ with $\alpha \in A_{L}$ and $\alpha=-\widetilde{\varepsilon}(\alpha)$ such that $\theta \in \mathcal{C}(\Lambda, 0, \alpha)$.

Proof Let $\varphi: A^{\prime} \rightarrow A^{\prime}$ be the involution given by conjugation by

$$
h=\left(\begin{array}{cc}
I_{M} & 0 \\
0 & -I_{M}
\end{array}\right) .
$$

Then $\left(\theta^{\prime}\right)^{\varphi}=\theta^{\prime}$, since it is trivial on the unipotent parts, and $\left(\theta^{\prime}\right)^{\varepsilon}=\theta^{\prime}$. Thus, $\theta^{\prime}$ is invariant under the subgroup $\Omega$ of $\operatorname{Aut}\left(G^{\prime}\right)$ generated by $\varepsilon$ and $\varphi$. As $\varepsilon \varphi=\varphi \varepsilon$, we see $\Omega \cong \mathbb{Z}_{2} \times \mathbb{Z}_{2}$, and thus by [22, Theorem 6.3] (see the Remarks at the bottom of page 139 there also), there is a choice of $\alpha$, fixed by $\varphi$ and with $\alpha=-\widetilde{\varepsilon}(\alpha)$, such that $\theta^{\prime} \in \mathcal{C}\left(\Lambda^{\prime}, 0, \alpha\right)$. Then $[\Lambda, n, 0, \alpha]$ is as required. 
Remark 1.4. Writing $\alpha=\operatorname{diag}\left(\alpha_{W}, 0,-\widetilde{\sigma}\left(\alpha_{W}\right)\right)$ for the element of Proposition 1.3 , we see that our original type $\left(J_{W}, \lambda_{W}\right)$ could also have been defined using $2 \alpha_{W}$ in place of $\beta$. Replacing $\beta$ by $2 \alpha_{W}$, the discussion following [3, 2.3 Theorem] applies in this situation (see especially [3, 2.3 Corollary] and the Remarks following). In particular, we may assume that $\sigma$ is such that $\widetilde{\sigma}$ induces a non-trivial galois involution on $E=F[\beta]$ which extends the involution $\mu$ on $F$ and sends $\beta$ to $-\beta$. From now on, we assume that $\sigma$ is chosen in this more careful way; this implies that $\sigma \varepsilon_{W}(\sigma) \in B_{W}$. Moreover, when $\mu$ is trivial, the degree $[E: F]$ is even so that $M=\operatorname{dim}_{F}(W)$ is even also.

As in the construction of the type $\left(J_{W}, \lambda_{W}\right)$, we will also need the transfer of $\theta$ to a minimal order, which we give now. Let $B^{\prime}$ denote the centralizer in $A^{\prime}$ of $E$ and put $\mathfrak{B}^{\prime}=\mathfrak{A}^{\prime} \cap B^{\prime}$, an $\mathfrak{o}_{E}$-order of period 2 with radical $\mathfrak{Q}^{\prime}$. Recall that we had, in $\S 1.1$, a minimal $\mathfrak{o}_{E}$-order $\mathfrak{B}_{W}^{m} \subset \mathfrak{B}_{W}$. Let $\mathfrak{B}^{\prime m} \subset \mathfrak{B}^{\prime}$ be the $\widetilde{\varepsilon}$-stable minimal $\mathfrak{o}_{E}$-order in $B^{\prime}$ given by

$$
\mathfrak{B}^{\prime m}=\left(\mathfrak{B}_{W}^{m} \oplus \widetilde{\varepsilon}\left(\mathfrak{B}_{W}^{m}\right)\right)+\mathfrak{Q}^{\prime},
$$

(cf. [3, 4.3]). Let $\mathcal{L}^{\prime \mathrm{m}}$ be the corresponding self-dual $\mathfrak{o}_{E}$-lattice chain in $V^{\prime}$, of $\mathfrak{o}_{E}$-period $2 r$, where $r=M /[E: F]$. Let $\Lambda^{\prime \mathrm{m}}$ be the self-dual $\mathfrak{o}_{E}$-lattice sequence in $V^{\prime}$ in which every lattice of $\mathcal{L}^{\prime \mathrm{m}}$ occurs twice and with the indexing chosen such that

$$
\Lambda^{\prime \mathrm{m}}(k)^{\#}=\Lambda^{\prime \mathrm{m}}(1-k), \quad \text { for all } k \in \mathbb{Z} .
$$

Note that $\left[\mathcal{L}^{\prime \mathrm{m}}, 2 n_{W}^{\mathrm{m}}, 0, \frac{1}{2} \beta\right]$ and $\left[\Lambda^{\prime \mathrm{m}}, 4 n_{W}^{\mathrm{m}}, 0, \frac{1}{2} \beta\right]$ are simple strata in $V^{\prime}$ whose associated groups and characters are the same up to a scaling of index.

Now let $\Lambda^{\mathrm{m}}$ be the $\mathfrak{o}_{F}$-lattice sequence in $V$ defined by

$$
\Lambda^{\mathrm{m}}(k)=\Lambda^{\prime \mathrm{m}}(k) \oplus \Lambda_{0}\left(\left\lceil\frac{k}{r}\right\rceil\right) .
$$

It is a self-dual lattice sequence of period re such that $\mathfrak{a}_{0}\left(\Lambda^{\mathrm{m}}\right) \subset \mathfrak{a}_{0}(\Lambda)$. Then $\left[\Lambda^{\mathrm{m}}, n^{\mathrm{m}}, 0, \frac{1}{2} \beta\right]$ is a semisimple stratum in $A$, where $n^{\mathrm{m}}=4 n_{W}^{\mathrm{m}}=n r$.

We put $\theta^{\prime \mathrm{m}}=\tau_{\Lambda^{\prime}, \Lambda^{\prime m}, \frac{1}{2} \beta}\left(\theta^{\prime}\right)$, a simple character of $H^{1}\left(\frac{1}{2} \beta, \Lambda^{\prime \mathrm{m}}\right)$; then also $\theta^{\prime \mathrm{m}}=\tau_{\mathfrak{A}_{W}^{\mathrm{m}}, \Lambda^{\prime \mathrm{m}}, \frac{1}{2} \beta}\left(\theta_{L}^{\mathrm{m}}\right)$. Finally, let $\theta^{\mathrm{m}}$ be the unique semisimple character of $H_{\mathrm{m}}^{1}=H^{1}\left(\frac{1}{2} \beta, \Lambda^{\mathrm{m}}\right)$ such that

$$
\left.\theta^{\mathrm{m}}\right|_{H^{1}\left(\frac{1}{2} \beta, \Lambda^{\prime m}\right)}=\theta^{\prime \mathrm{m}}
$$

In the language of [24, §3.5], we have $\theta^{\mathrm{m}}=\tau_{\Lambda, \Lambda^{\mathrm{m}}, \frac{1}{2} \beta}(\theta)$.

\section{Covers and Hecke Algebras}

In this chapter, we construct our cover and find the structure of its Hecke algebra. The method of construction of the cover is (apart from the added complication of the anisotropic part) the same as that in [3] (the reader may find it helpful to consult the diagram on page 552 of [3], especially the left side). However, here we obtain full information on the intertwining of the cover (rather than just a bound), and it is this which allows us, in $\$ 2.3$, to identify the Hecke algebra. In order to obtain this information on the intertwining, we need to review carefully the construction and keep track of the intertwining at each stage. 
We continue with the notation above, so we have $\left[\Lambda, n, 0, \frac{1}{2} \beta\right]$ a skew semisimple stratum in $A$, with $\beta \in A_{L}$, and $\theta \in \mathcal{C}\left(\Lambda, 0, \frac{1}{2} \beta\right)$ such that

$$
\left.\theta\right|_{\bar{H}^{1}}=\widetilde{i\left(\theta_{W}\right)}
$$

We also have $E=F[\beta]$ and $B$ the $A$-centralizer of $E$; note here that, with notation as above, $B^{\times}=\left(B^{\prime}\right)^{\times} \times G_{0}$. From Remark 1.4, the galois involution $\mu$ extends to $E$ (as $\widetilde{\sigma}$ when we consider $E$ embedded in $A_{W}$, or as $\widetilde{\varepsilon}$ when we consider $E$ embedded in $A$ ), and we write $E_{0}$ for the fixed subfield, which has index 2. We fix a uniformizer $\varpi_{E}$ of $E$ such that $\varpi_{E}{ }^{\mu}= \pm \varpi_{E}$. Note that $\beta \in A_{W}$ embeds in $A$ as $\operatorname{diag}(\beta, 0,-\widetilde{\sigma}(\beta))=\operatorname{diag}(\beta, 0, \beta)$.

\section{$2.1 \quad$ G-Covers}

We begin by doing some work in $G$, before using Glauberman's correspondence to transfer this information to $\bar{G}$.

Recall that, given $\rho$ a representation of a subgroup $H$ of $G$ and $g \in G$, the intertwining space $I_{g}(\rho \mid H)$ is

$$
I_{g}(\rho \mid H)=\operatorname{Hom}_{H \cap{ }^{g} H}\left(\rho,{ }^{g} \rho\right),
$$

where ${ }^{g} H=g H g^{-1}$ and ${ }^{g} \rho$ is the representation of ${ }^{g} H$ given by ${ }^{g} \rho\left(g h g^{-1}\right)=\rho(h)$, for $h \in H$. The $G$-intertwining $I_{G}(\rho \mid H)$ of $\rho$ is then defined to be

$$
I_{G}(\rho)=I_{G}(\rho \mid H)=\left\{g \in G: I_{g}(\rho \mid H) \neq 0\right\} .
$$

In our situation, we recall the following, from [22, Theorem 3.14, Corollary 4.2 and Proposition 4.3]:

Lemma 2.1. (i) $I_{G}(\theta)=J^{1} B^{\times} J^{1}$.

(ii) There exists a unique irreducible representation $\eta$ of $J^{1}$ which contains $\theta$. Moreover, $\operatorname{dim} \eta=$ $\left(J^{1}: H^{1}\right)^{\frac{1}{2}}$ and, for $g \in G$,

$$
\operatorname{dim} I_{g}\left(\eta \mid J^{1}\right)= \begin{cases}1 & \text { if } g \in J^{1} B^{\times} J^{1} \\ 0 & \text { otherwise. }\end{cases}
$$

From [6, §7.1] (together with [22, §3.3]), $H^{1}$ has an Iwahori decomposition with respect to $(L, P)$ and

$$
H^{1} \cap L=H^{1}\left(\frac{1}{2} \beta, \mathfrak{A}_{W}\right) \times U^{1}\left(\Lambda_{0}\right) \times H^{1}\left(\frac{1}{2} \beta, \mathfrak{A}_{W}\right) .
$$

There are also similar decompositions for $J^{1}$ and for $J$. Moreover, $\theta$ is trivial on $H^{1} \cap U$ and $H^{1} \cap U_{-}$, and the restriction of $\theta$ to $H^{1} \cap L$ takes the form

$$
\left.\theta\right|_{H^{1} \cap L}=\theta_{L} \otimes \mathbf{1} \otimes \theta_{L}
$$

We recall ([22, Proposition 4.1] - see also [6, §3.4]) that the pairing

$$
\left(j, j^{\prime}\right) \mapsto \theta\left[j, j^{\prime}\right], \quad \text { for } j, j^{\prime} \in J^{1}
$$

induces a nondegenerate alternating bilinear form $\boldsymbol{k}_{\theta}$ on $J^{1} / H^{1}$; likewise, we have a nondegenerate alternating bilinear form $\boldsymbol{k}_{\theta_{L}}$ on $J^{1}\left(\frac{1}{2} \beta, \mathfrak{A}_{W}\right) / H^{1}\left(\frac{1}{2} \beta, \mathfrak{A}_{W}\right)$. Then, exactly as in [6. Proposition 7.2.3], we get: 
Lemma 2.2 (cf. [6, Proposition 7.2.3]). (i) The subspaces $J^{1} \cap U_{-} / H^{1} \cap U_{-}$and $J^{1} \cap U / H^{1} \cap U$ of $J^{1} / H^{1}$ are both totally isotropic for the form $\boldsymbol{k}_{\theta}$ and orthogonal to the subspace $J^{1} \cap L / H^{1} \cap L$.

(ii) The restriction of $\boldsymbol{k}_{\theta}$ to the group

$$
J^{1} \cap L / H^{1} \cap L=J^{1}\left(\frac{1}{2} \beta, \mathfrak{A}_{W}\right) / H^{1}\left(\frac{1}{2} \beta, \mathfrak{A}_{W}\right) \times J^{1}\left(\frac{1}{2} \beta, \mathfrak{A}_{W}\right) / H^{1}\left(\frac{1}{2} \beta, \mathfrak{A}_{W}\right)
$$

is the orthogonal sum of the pairings $\boldsymbol{k}_{\theta_{L}}, \boldsymbol{k}_{\theta_{L}}$.

(iii) We have an orthogonal sum decomposition

$$
\frac{J^{1}}{H^{1}}=\frac{J^{1} \cap L}{H^{1} \cap L} \perp\left(\frac{J^{1} \cap U_{-}}{H^{1} \cap U_{-}} \times \frac{J^{1} \cap U}{H^{1} \cap U}\right)
$$

We define the groups

$$
H_{P}^{1}=H^{1}\left(J^{1} \cap U\right), \quad J_{P}^{1}=H^{1}\left(J^{1} \cap P\right), \quad J_{P}=H^{1}(J \cap P) .
$$

Since $J^{1}$ normalizes $\theta$ and $\left.\theta\right|_{H^{1} \cap U}$ is trivial, we can define the character $\theta_{P}$ of $H_{P}^{1}$ by

$$
\theta_{P}(h u)=\theta(h), \quad \text { for } h \in H^{1}, u \in J^{1} \cap U .
$$

As in [6, §7.2], we immediately get:

Corollary 2.3 (cf. [6, Propositions 7.2.4, 7.2.9]). There is a unique irreducible representation $\eta_{P}$ of $J_{P}^{1}$ such that $\left.\eta_{P}\right|_{H_{P}^{1}}$ contains $\theta_{P}$. Moreover, $\eta \simeq \operatorname{Ind}_{J_{P}^{1}}^{J^{1}} \eta_{P}$ and, for each $b \in B^{\times}$, there is a unique $\left(J_{P}^{1}, J_{P}^{1}\right)$-double coset in $J^{1} b J^{1}$ which intertwines $\eta_{P}$.

We note also that we certainly have $\left.\eta_{P}\right|_{J_{P}^{1} \cap L} \simeq \eta_{L} \otimes \mathbf{1} \otimes \eta_{L}$.

Proposition 2.4. We have $I_{G}\left(\theta_{P}\right)=J_{P}^{1} B^{\times} J_{P}^{1}$ and hence $I_{G}\left(\eta_{P}\right)=J_{P}^{1} B^{\times} J_{P}^{1}$.

Proof This is a special case of [25, Lemma 5.8].

We will also need similar results for our character $\theta^{\mathrm{m}}$ of $H_{\mathrm{m}}^{1}=H^{1}\left(\frac{1}{2} \beta, \Lambda^{\mathrm{m}}\right)$. Let $\eta^{\mathrm{m}}$ be the unique irreducible representation of $J_{\mathrm{m}}^{1}=J^{1}\left(\frac{1}{2} \beta, \Lambda^{\mathrm{m}}\right)$ which contains $\theta^{\mathrm{m}}$. As above, $H_{\mathrm{m}}^{1}$ and $J_{\mathrm{m}}^{1}$ have Iwahori decompositions with respect to $(L, P)$ and we can define the character $\theta_{P}^{\mathrm{m}}$ of $H_{\mathrm{m}, P}^{1}=$ $H_{\mathrm{m}}^{1}\left(J_{\mathrm{m}}^{1} \cap U\right)$ by trivial extension of $\theta^{\mathrm{m}}$. The same proofs (indeed, they are somewhat simpler) as those of Lemma 2.2. Corollary 2.3 and Proposition 2.4 show that

$$
I_{G}\left(\theta_{P}^{\mathrm{m}}\right)=J_{\mathrm{m}, P}^{1} B^{\times} J_{\mathrm{m}, P}^{1},
$$

where $J_{\mathrm{m}, P}^{1}=H_{\mathrm{m}}^{1}\left(J_{\mathrm{m}}^{1} \cap P\right)$, that there is a unique irreducible representation $\eta_{P}^{\mathrm{m}}$ of $J_{\mathrm{m}, P}^{1}$ which contains $\theta_{P}^{\mathrm{m}}$, and that $\eta^{\mathrm{m}}=\operatorname{Ind}_{J_{\mathrm{m}, P}^{1}}^{J_{1}^{1}} \eta_{P}^{\mathrm{m}}$.

Let $\theta_{L}^{\mathrm{m}}$ denote the transfer to $H^{1}\left(\frac{1}{2} \beta, \mathfrak{A}_{W}^{\mathrm{m}}\right)$ of $\theta_{L}$ (this is just the restriction of $\theta^{\mathrm{m}}$ to one of the copies of $\left.G_{W} \subset L\right)$. Then, if $\eta_{L}^{\mathrm{m}}$ denotes the unique irreducible representation of $J^{1}\left(\frac{1}{2} \beta, \mathfrak{A}_{W}^{\mathrm{m}}\right)$ which contains $\theta_{L}^{\mathrm{m}}$, we have

$$
\left.\eta_{P}^{\mathrm{m}}\right|_{H_{\mathrm{m}}^{1} \cap L} \simeq \eta_{L}^{\mathrm{m}} \otimes \mathbf{1} \otimes \eta_{L}^{\mathrm{m}}
$$




\section{$2.2 \overline{\mathrm{G}}$-Covers}

Now we will transfer the information obtained in the last section to $\bar{G}$, using Glauberman's correspondence (see [10, or 22] for the situation here). Let $\Omega$ denote a 2-group of automorphisms of $G$. Recall that if $H$ is a pro- $p$ subgroup of $G$ and $H^{\Omega}$ is the group of $\Omega$-fixed points, then there is a bijection, denoted $\rho \leftrightarrow \boldsymbol{g}_{\Omega}(\rho)$ between (equivalence classes of) irreducible representations of $H$ with $\rho^{\omega} \simeq \rho$, for all $\omega \in \Omega$, and (equivalence classes of) irreducible representations of $H^{\Omega}$. Further, this correspondence commutes with irreducible restriction and irreducible induction. Recall also that the representation $\boldsymbol{g}_{\Omega}(\rho)$ is characterized as the unique component of $\left.\rho\right|_{H^{\Omega}}$ appearing with odd multiplicity.

We will usually apply this correspondence with $\Omega$ the group of automorphisms of $G$ consisting of $\varepsilon$ and the identity, in which case we will just write $\boldsymbol{g}$ for the correspondence. Note also that, for $\varepsilon$-stable pro- $p$ subgroups $H$ of $G$, we have $H^{\varepsilon}=\bar{H}^{+}=\bar{H}$.

We write $\bar{\theta}=\boldsymbol{g}(\theta)=\left.\theta\right|_{\bar{H}^{1}}$, a skew semisimple character (see [22, §3.4]). We also set

$$
\bar{G}_{E}^{+}=B \cap \bar{G}^{+} \text {and } \bar{G}_{E}=B \cap \bar{G}
$$

so that $\bar{G}_{E}^{+}$(respectively $\bar{G}_{E}$ ) is the direct product of a unitary group, for the quadratic extension $E / E_{0}$, and the anisotropic group $\bar{G}_{0}^{+}$(respectively $\bar{G}_{0}$ ).

We recall first the following, from [22, Theorem 3.16] (see also the Remarks following op. cit. Corollary 4.2), and [24, Proposition 3.3.1]:

Lemma 2.5. (i) $I_{\bar{G}^{+}}(\bar{\theta})=\bar{J}^{1} \bar{G}_{E}^{+} \bar{J}^{1}$ and $I_{\bar{G}}(\bar{\theta})=\bar{J}^{1} \bar{G}_{E} \bar{J}^{1}$.

(ii) There exists a unique irreducible representation $\bar{\eta}$ of $\bar{J}^{1}$ which contains $\bar{\theta}$. Moreover, $\bar{\eta}=\boldsymbol{g}(\eta)$, $\operatorname{dim} \bar{\eta}=\left(\bar{J}^{1}: \bar{H}^{1}\right)^{\frac{1}{2}}$ and, for $g \in \bar{G}^{+}$,

$$
\operatorname{dim} I_{g}\left(\bar{\eta} \mid \bar{J}^{1}\right)= \begin{cases}1 & \text { if } g \in \bar{J}^{1} \bar{G}_{E}^{+} \bar{J}^{1} \\ 0 & \text { otherwise. }\end{cases}
$$

We also put $\bar{\theta}_{P}=\boldsymbol{g}\left(\theta_{P}\right)=\left.\theta_{P}\right|_{H_{P}^{1}}$. Note that we have

$$
\bar{\theta}_{P}=\widetilde{i\left(\theta_{W}\right)}
$$

Remark 2.6. (i) Note that, in the language of [25, Definition 5.1], the decomposition $V=$ $W \oplus V_{0} \oplus W$ (of which the Levi subgroup $L$ is the stabilizer) is properly subordinate to the stratum $[\Lambda, n, 0, \beta]$. Then [25, Lemma 5.3] implies that $U(\Lambda) \cap B \cap U=U^{1}(\Lambda) \cap B \cap U$. In particular, $\bar{J} \cap \bar{U}=\bar{J}^{1} \cap \bar{U}$.

(ii) From (i), we have $\bar{J}_{P} \cap \bar{U}=\bar{H}_{P}^{1} \cap \bar{U}$, and the same is true for $\bar{U}_{-}$. Then the property $\bar{\theta}_{P}=\widetilde{i\left(\theta_{W}\right)}$ implies (see [2, Lemma 1(ii)]) that

$$
\left(\bar{J}_{P} \cap \bar{U}\right)\left(\bar{J}_{P} \cap \bar{U}_{-}\right) \subset\left(\bar{J}_{P} \cap \bar{U}_{-}\right) \operatorname{Ker}\left(i\left(\theta_{W}\right)\right)\left(\bar{J}_{P} \cap \bar{U}\right) .
$$

In particular (by loc. cit.), if $\rho$ is a representation of a subgroup $K$ of $J_{W} \times \bar{G}_{0}$ which restricts to a multiple of $\theta_{W}$, then $\widetilde{i(\rho)}$ is a well-defined representation of $\left(\bar{J}_{P} \cap \bar{U}_{-}\right)(i(K))\left(\bar{J}_{P} \cap \bar{U}\right)$. We will use this several times, for example, in the definitions of $\widetilde{\eta}_{P}$ and $\bar{\kappa}_{P}$ below. 
From Proposition 2.4, together with [22, Corollary 2.5] and[23, Theorem 2.3], we get:

Proposition 2.7. $I_{\bar{G}^{+}}\left(\bar{\theta}_{P}\right)=\bar{J}_{P}^{1} \bar{G}_{E}^{+} \bar{J}_{P}^{1}$ and $I_{\bar{G}}\left(\bar{\theta}_{P}\right)=\bar{J}_{P}^{1} \bar{G}_{E} \bar{J}_{P}^{1}$.

Let $\bar{\theta}^{\mathrm{m}}=\boldsymbol{g}\left(\theta^{\mathrm{m}}\right)=\left.\theta^{\mathrm{m}}\right|_{\bar{H}_{\mathrm{m}}^{1}}$. Let $\bar{\eta}$ be the unique irreducible representation of $\overline{\boldsymbol{J}}^{1}$ which contains $\bar{\theta}$, as in Lemma 2.5, and let $\bar{\eta}^{\mathrm{m}}$ be the unique irreducible representation of $\bar{J}_{\mathrm{m}}^{1}$ which contains $\bar{\theta}^{\mathrm{m}}$. Note that $\bar{\theta}_{P}=\boldsymbol{g}\left(\theta_{P}\right)$ is the trivial extension of $\bar{\theta}$ to $\bar{H}_{P}^{1}$ and, similarly, $\bar{\theta}_{P}^{\mathrm{m}}=\boldsymbol{g}\left(\theta_{P}^{\mathrm{m}}\right)$ the trivial extension of $\bar{\theta}^{\mathrm{m}}$ to $\bar{H}_{\mathrm{m}, P}^{1}$.

We also put $\bar{\eta}_{P}=\boldsymbol{g}\left(\eta_{P}\right)$ and $\bar{\eta}_{P}^{\mathrm{m}}=\boldsymbol{g}\left(\eta_{P}^{\mathrm{m}}\right)$. By taking $\varepsilon$-fixed points in $J^{1} / H^{1}$, we can imitate Lemma 2.2 and Corollary 2.3 to show that there is a unique irreducible representation of $\bar{J}_{P}^{1}$ which contains $\theta_{P}$ and, since $\eta_{P}{\overline{H_{P}}}_{P}^{1}$ is a multiple of $\bar{\theta}_{P}$, we see that this must be $\bar{\eta}_{P}$. Likewise, $\bar{\eta}_{P}^{\mathrm{m}}$ is the unique irreducible representation of $\bar{J}_{\mathrm{m}, P}^{1}$ containing $\bar{\theta}_{P}^{\mathrm{m}}$. We also have

$$
\bar{\eta}_{P}=\widetilde{i\left(\eta_{W}\right)} \quad \text { and } \quad \bar{\eta}_{P}^{\mathrm{m}}=\widetilde{i\left(\eta_{W}^{\mathrm{m}}\right)}
$$

since the representations on the right restrict to multiples of $\widetilde{i\left(\theta_{W}\right)}=\bar{\theta}_{P}$ and $\widetilde{i\left(\theta_{W}^{\mathrm{m}}\right)}=\bar{\theta}_{P}^{\mathrm{m}}$ respectively.

The group $U(\Lambda) \cap B$ has Iwahori decomposition with respect to $(L, P)$, and normalizes $J^{1}\left(\frac{1}{2} \beta, \Lambda\right)$ and $H^{1}\left(\frac{1}{2} \beta, \Lambda\right)$. Hence $U(\Lambda) \cap B$ normalizes $J_{P}^{1}$ and, since $U\left(\Lambda^{\mathrm{m}}\right)$ is contained in $U(\Lambda)$, we can form the group $\widetilde{J}_{P}^{1}=\left(\bar{U}^{1}\left(\Lambda^{\mathrm{m}}\right) \cap \bar{G}_{E}\right) \bar{J}_{P}^{1}$. We also recall that we have the representation $\widetilde{\eta}_{W}$ of $\widetilde{J}_{W}^{1}=U^{1}\left(\mathfrak{B}_{W}^{m}\right) J_{W}^{1}$ (see 1.1$)$ and observe that $\widetilde{J}_{P}^{1} \cap \bar{L}=i\left(\widetilde{J}_{W}^{1} \times \bar{U}^{1}\left(\Lambda_{0}\right)\right)$.

Proposition 2.8 (cf. [6, Propositions 5.1.15, 5.1.19]). There is a unique representation $\widetilde{\eta}_{P}$ of $\widetilde{J}_{P}^{1}$ such that

(i) $\left.\widetilde{\eta}_{P}\right|_{\bar{J}_{P}^{1}}=\bar{\eta}_{P}$;

(ii) $\widetilde{\eta}_{P}$ and $\bar{\eta}_{P}^{\mathrm{m}}$ induce equivalent irreducible representations of $\bar{U}^{1}\left(\Lambda^{\mathrm{m}}\right)$.

Moreover, $\widetilde{\eta}_{P}=\widetilde{i\left(\widetilde{\eta}_{W}\right)}$ and

$$
\operatorname{dim} I_{g}\left(\widetilde{\eta}_{P}\right)= \begin{cases}1 & \text { if } g \in \widetilde{J}_{P}^{1} \bar{G}_{E}^{+} \widetilde{J}_{P}^{1} \\ 0 & \text { otherwise }\end{cases}
$$

Proof Let $\Omega_{1}$ be the group of automorphisms of $\bar{G}^{+}$generated by conjugation by

$$
h_{1}=\left(\begin{array}{ccc}
-I_{M} & & \\
& I_{D} & \\
& & -I_{M}
\end{array}\right) .
$$

Then $\left(\bar{G}^{+}\right)^{\Omega_{1}}=\left(\bar{G}^{\prime}\right)^{+} \times \bar{G}_{0}^{+}$. We will denote the Glauberman correspondence $\boldsymbol{g}_{\Omega_{1}}$ on representations of pro- $p$ subgroups of $\bar{G}$ by $\boldsymbol{g}_{1}$. We note that, since all our representations are trivial on $U^{\prime}$ and $U^{\prime-}$, they are fixed by $\Omega_{1}$ and, moreover, $\boldsymbol{g}_{1}$ is just restriction for these representations (since their restrictions are irreducible). 
Let $\Omega_{2}$ be the group of automorphisms of $\left(\bar{G}^{+}\right)^{\Omega_{1}}$ generated by conjugation by

$$
h_{2}=\left(\begin{array}{ccc}
I_{M} & & \\
& I_{D} & \\
& & -I_{M}
\end{array}\right) \text {. }
$$

Then $\left(\left(\bar{G}^{+}\right)^{\Omega_{1}}\right)^{\Omega_{2}}=\bar{L}$. We will denote the Glauberman correspondence $\boldsymbol{g}_{\Omega_{2}}$ on representations of pro- $p$ subgroups of $\left(\bar{G}^{+}\right)^{\Omega_{1}}$ by $\boldsymbol{g}_{2}$. As above, since all our representations are trivial on $U$ and $U^{-}$, their transfers to $\left(\bar{G}^{+}\right)^{\Omega_{1}}$ are fixed by $\Omega_{2}$ and, moreover, $\boldsymbol{g}_{2}$ is again just restriction for these representations (since their restrictions are still irreducible).

We write $\boldsymbol{g}_{\Omega}$ for the composition $\boldsymbol{g}_{2} \circ \boldsymbol{g}_{1}$. Then we have

$$
\boldsymbol{g}_{\Omega}\left(\bar{\eta}_{P}\right)=i\left(\eta_{W}\right) \quad \text { and } \quad \boldsymbol{g}_{\Omega}\left(\bar{\eta}_{P}^{\mathrm{m}}\right)=i\left(\eta_{W}^{\mathrm{m}}\right) .
$$

We define $\widetilde{\eta}_{P}$ to be $\widetilde{i\left(\widetilde{\eta}_{W}\right)}$. Then we clearly have $\boldsymbol{g}_{\Omega}\left(\widetilde{\eta}_{P}\right)=i\left(\widetilde{\eta}_{W}\right)$ and $\left.\widetilde{\eta}_{P}\right|_{\bar{J}_{P}^{1}}=\bar{\eta}_{P}$. Also, since the intertwining of $\widetilde{\eta}_{P}$ is contained in that of $\bar{\eta}_{P}$,

$$
I_{\bar{G}^{+}}\left(\widetilde{\eta}_{P}\right) \cap \bar{U}^{1}\left(\Lambda^{\mathrm{m}}\right) \subset \bar{J}_{P}^{1} \bar{G}_{E}^{+} \bar{J}_{P}^{1} \cap \bar{U}^{1}\left(\Lambda^{\mathrm{m}}\right)=\left(\bar{U}^{1}\left(\Lambda^{\mathrm{m}}\right) \cap \bar{G}_{E}\right) \bar{J}_{P}^{1}=\widetilde{J}_{P}^{1},
$$

so the induced representation $\operatorname{Ind}_{\widetilde{J}_{P}^{1}}^{\bar{U}^{1}\left(\Lambda^{\mathrm{m}}\right)} \widetilde{\eta}_{P}$ is irreducible. Likewise, $\operatorname{Ind}_{\bar{J}_{\mathrm{m}, P}^{1}\left(\Lambda^{\mathrm{m}}\right)}^{\bar{\eta}_{P}^{\mathrm{m}}}$ is irreducible. Then, since Glauberman's correspondence commutes with irreducible induction, we have

$$
\begin{aligned}
\boldsymbol{g}_{\Omega}\left(\operatorname{Ind}_{\widetilde{J}_{P}^{1}}^{\bar{U}^{1}\left(\Lambda^{\mathrm{m}}\right)} \widetilde{\eta}_{P}\right) & =\operatorname{Ind}_{i\left(\widetilde{J}_{W}^{1} \times \bar{U}^{1}\left(\Lambda_{0}\right)\right)}^{i\left(U^{1}\left(\Lambda_{0}^{\mathrm{m}}\right) \times \bar{U}^{1}\left(\Lambda_{0}\right)\right)} i\left(\widetilde{\eta}_{W}\right) \\
& \simeq \operatorname{Ind}_{i\left(J^{1}\left(\beta, \mathfrak{A}_{W}^{\mathrm{m}}\right) \times \bar{U}^{1}\left(\Lambda_{0}\right)\right)}^{i\left(U^{1}\left(\Lambda^{\mathrm{m}}\right) \times \bar{U}^{1}\left(\Lambda_{0}\right)\right)} i\left(\eta_{W}^{\mathrm{m}}\right)=\boldsymbol{g}_{\Omega}\left(\operatorname{Ind}_{\bar{J}_{\mathrm{m}, P}^{1}}^{\bar{U}^{1}\left(\Lambda^{\mathrm{m}}\right)} \bar{\eta}_{P}^{\mathrm{m}}\right) .
\end{aligned}
$$

Condition (ii) now follows as $\boldsymbol{g}_{\Omega}$ is injective.

Now we show that these two conditions determine $\widetilde{\eta}_{P}$ uniquely. For this, we need only show that $\bar{\eta}_{P}$

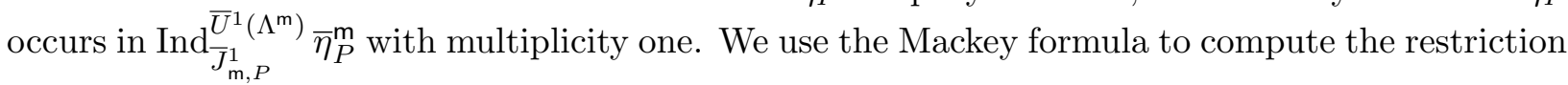

$$
\operatorname{Res}_{\bar{J}_{P}^{1}}^{\bar{U}^{1}\left(\Lambda^{\mathrm{m}}\right)} \operatorname{Ind}_{\widetilde{J}_{P}^{1}}^{\bar{U}^{1}\left(\Lambda^{\mathrm{m}}\right)} \widetilde{\eta}_{P}
$$

If $x \in \bar{U}^{1}\left(\Lambda^{\mathrm{m}}\right)$ intertwines $\widetilde{\eta}_{P}$ with $\bar{\eta}_{P}$, then it intertwines $\bar{\eta}_{P}$ with itself so lies in $\widetilde{J}_{P}^{1}$, as above. Thus the multiplicity of $\bar{\eta}_{P}$ in $\operatorname{Ind}_{\bar{J}_{\mathrm{m}, P}^{1}}{\overline{\Lambda^{1}}}^{\mathrm{m})} \bar{\eta}_{P}^{\mathrm{m}}$ is equal to its multiplicity in $\widetilde{\eta}_{P}$, which we know to be one.

Finally, we must show that all of $\bar{G}_{E}^{+}$intertwines $\widetilde{\eta}_{P}$. So suppose $b \in \bar{G}_{E}^{+}$. Since $b$ intertwines $\bar{\eta}_{P}^{\mathrm{m}}$, it certainly intertwines

$$
\operatorname{Ind}_{\widetilde{J}_{P}^{1}}^{\bar{U}^{1}\left(\Lambda^{\mathrm{m}}\right)} \widetilde{\eta}_{P} \simeq \operatorname{Ind}_{\bar{J}_{\mathbf{m}, P}^{1}}^{\bar{U}^{1}\left(\Lambda^{\mathrm{m}}\right)} \bar{\eta}_{P}^{\mathrm{m}}
$$

We deduce that there exist $u, v \in \bar{U}^{1}\left(\Lambda^{\mathrm{m}}\right)$ such that $u b v$ intertwines $\widetilde{\eta}_{P}$. In particular, $u b v$ intertwines $\bar{\eta}_{P}$ so there exist $j_{1}, j_{2} \in \bar{J}_{P}^{1}$ such that $j_{1} u b v j_{2} \in \bar{G}_{E}^{+}$. Note that this element also still intertwines $\widetilde{\eta}_{P}$.

Now

$$
\begin{aligned}
U^{1}\left(\Lambda^{\mathrm{m}}\right) b U^{1}\left(\Lambda^{\mathrm{m}}\right) \cap B^{\times} & =U^{1}\left(\Lambda^{\mathrm{m}}\right) b U^{1}\left(\Lambda^{\mathrm{m}}\right) \cap L^{\prime} \cap B^{\times} \\
& =\left(U^{1}\left(\Lambda^{\mathrm{m}}\right) \cap L^{\prime}\right) b\left(U^{1}\left(\Lambda^{\mathrm{m}}\right) \cap L^{\prime}\right) \cap B^{\times}
\end{aligned}
$$


by [23, Lemma 2.1] applied with $\Gamma=\Omega_{1}$. Further,

$$
\left(U^{1}\left(\Lambda^{\mathrm{m}}\right) \cap L^{\prime}\right) b\left(U^{1}\left(\Lambda^{\mathrm{m}}\right) \cap L^{\prime}\right) \cap B^{\times}=\left(U^{1}\left(\Lambda^{\mathrm{m}}\right) \cap B^{\times}\right) b\left(U^{1}\left(\Lambda^{\mathrm{m}}\right) \cap B^{\times}\right),
$$

by [6, Theorem 1.6.1] (which we may apply since, in $A^{\prime}$ we have a simple stratum). Then, applying [23, Lemma 2.1] again, with $\Gamma=\{1, \varepsilon\}$, we get

$$
\bar{U}^{1}\left(\Lambda^{\mathrm{m}}\right) b \bar{U}^{1}\left(\Lambda^{\mathrm{m}}\right) \cap \bar{G}_{E}^{+}=\left(\bar{U}^{1}\left(\Lambda^{\mathrm{m}}\right) \cap \bar{G}_{E}\right) b\left(\bar{U}^{1}\left(\Lambda^{\mathrm{m}}\right) \cap \bar{G}_{E}\right) .
$$

So there exist $u^{\prime}, v^{\prime} \in \bar{U}^{1}\left(\Lambda^{\mathrm{m}}\right) \cap \bar{G}_{E}$ such that $u^{\prime} b v^{\prime}=j_{1} u b v j_{2}$ and, since $u^{\prime}, v^{\prime} \in \widetilde{J}_{P}^{1}$, we see that $b$ intertwines $\widetilde{\eta}_{P}$.

Finally, the assertion that the intertwining spaces are all 1-dimensional follows immediately from Lemma 2.5(ii), since $\widetilde{\eta}_{P}$ extends $\bar{\eta}$.

Now we define $\bar{\kappa}_{P}=\widetilde{i\left(\kappa_{W}\right)}$, a representation of $\bar{J}_{P}$, so that $\left.\bar{\kappa}_{P}\right|_{\widetilde{J}_{P}^{1}} \simeq \widetilde{\eta}_{P}$. We also put $\bar{\rho}_{P}=\widetilde{i\left(\rho_{W}\right)}$, a representation of $\bar{J}_{P}$ trivial on $\bar{J}_{P}^{1}$, and

$$
\bar{\lambda}_{P}=\widetilde{i\left(\lambda_{W}\right)}=\bar{\kappa}_{P} \otimes \bar{\rho}_{P} .
$$

Writing matrices with respect to the (non-Witt) basis $\mathcal{B} \cup \mathcal{B}_{0} \cup \mathcal{B}$ of $V=W \oplus V_{0} \oplus W$, we set

$$
w_{1}=\left(\begin{array}{ccc} 
& & I_{M} \\
& I_{D} & \\
\nu \sigma \varepsilon_{W}(\sigma) & &
\end{array}\right),
$$

where $I_{k}$ denotes the $k \times k$ identity matrix. We also put

$$
\overline{\mathrm{W}}=\left\{i\left(\varpi_{E}^{a}, \mathbf{1}_{V_{0}}\right): a \in \mathbb{Z}\right\} \cup\left\{i\left(\varpi_{E}^{a}, \mathbf{1}_{V_{0}}\right) w_{1}: a \in \mathbb{Z}\right\},
$$

where $\varpi_{E}$ is our fixed uniformizer of $E \subset A_{W}$ and $\mathbf{1}_{V_{0}}$ is the identity map on $V_{0}$.

Proposition 2.9. $I_{\bar{G}}\left(\bar{\lambda}_{P}\right)=\bar{J}_{P} \overline{\mathrm{W}} \bar{J}_{P}$.

Proof Since $\bar{J}_{P} \cap \bar{L}=i\left(J_{W}\right)$, while $\varpi_{E}$ and $\sigma \varepsilon_{W}(\sigma)$ normalize $J_{W}$, the elements of $\overline{\mathrm{W}}$ normalize $\bar{J}_{P} \cap \bar{L}$. Since $\varpi_{E}$ normalizes $\lambda_{W}$ and $\lambda_{W} \simeq \lambda_{W} \circ \widetilde{\sigma}$ (see Proposition 1.1), we see that the elements of $\overline{\mathrm{W}}$ normalize $i\left(\lambda_{W}\right)$. On the other hand, they either preserve $\bar{U}$ and $\bar{U}_{-}$or interchange them so we see that every element of $\overline{\mathrm{W}}$ intertwines $\bar{\lambda}_{P}=\widetilde{i\left(\lambda_{W}\right)}$. Hence we have $I_{\bar{G}}\left(\bar{\lambda}_{P}\right) \supset \bar{J}_{P} \overline{\mathrm{W}} \bar{J}_{P}$.

The proof of the opposite containment, which is a variant of the proof of [6, Proposition 5.3.2], is inspired by [3, page 551]; in place of [3, 4.2 Lemma], we use [25, Proposition 1.1], which is a slight generalization of [18, Proposition 4.13]. It is almost identical to the proof of [25, Proposition 6.14], except that the definition of $\bar{\kappa}_{P}$ there is a priori slightly different.

Suppose $g \in \bar{G}$ intertwines $\bar{\lambda}_{P}=\bar{\kappa}_{P} \otimes \bar{\rho}_{P}$, so that $g \in I_{\bar{G}}\left(\bar{\eta}_{P} \mid \bar{J}_{P}^{1}\right)=\bar{J}_{P} \bar{G}_{E} \bar{J}_{P}$, as $\bar{\rho}_{P}$ is trivial on $\bar{J}_{P}^{1}$. Thus, we may assume $g$ lies in $\bar{G}_{E}$. Moreover $\bar{J}_{P} \cap \bar{G}_{E}=\bar{U}(\Lambda) \cap \bar{G}_{E}$ is a parahoric subgroup of $\bar{G}_{E}$ containing the Iwahori subgroup $\bar{U}\left(\Lambda^{\mathrm{m}}\right) \cap \bar{G}_{E}$. Therefore, we may further assume $g$ is a distinguished double coset representative for $\bar{U}(\Lambda) \cap \bar{G}_{E} \backslash \bar{G}_{E} / \bar{U}(\Lambda) \cap \bar{G}_{E}$ (see [18, §3] or [25, §1] for this notion). 
Since $\operatorname{dim} I_{g}\left(\bar{\eta}_{P}, \bar{J}_{P}^{1}\right)=1$, we can imitate the proof of [6, Proposition 5.3.2] to get that any non-zero intertwining operator in $I_{g}\left(\bar{\lambda}_{P}, \bar{J}_{P}\right)$ has the form $S \otimes T$, with $S \in I_{g}\left(\bar{\eta}_{P}, \bar{J}_{P}^{1}\right)$ and $T$ an endomorphism of the space of $\bar{\rho}_{P}$. Now the operator $S$ also intertwines the restriction $\left.\kappa_{P}\right|_{\widetilde{J}_{P}^{1}}=\widetilde{\eta}_{P}$ so, again as in $\underline{6}$, Proposition 5.3.2], it follows that $T$ belongs to $I_{g}\left(\bar{\rho}_{P} \mid \widetilde{J}_{P}^{1}\right)$. In particular, $g$ intertwines $\bar{\rho}_{P} \mid \widetilde{J}_{P}^{1} \cap \bar{G}_{E}$. But $\widetilde{J}_{P}^{1} \cap \bar{G}_{E}=\bar{U}^{1}\left(\Lambda^{\mathrm{m}}\right) \cap \bar{G}_{E}$ is the radical of the Iwahori subgroup $\bar{U}\left(\Lambda^{\mathrm{m}}\right) \cap \bar{G}_{E}$ of $\bar{G}_{E}$ contained in $\bar{U}(\Lambda) \cap \bar{G}_{E}$. By [25, Proposition 1.1] and the Remarks that follow it, we conclude that we can assume that $g$ normalizes $\bar{U}(\Lambda) \cap \bar{G}_{E} \cap \bar{L}$.

Finally, we notice (cf. [3, 4.1]) that $N_{\bar{G}_{E}}\left(\bar{U}(\Lambda) \cap \bar{G}_{E} \cap \bar{L}\right)=\left(\bar{U}(\Lambda) \cap \bar{G}_{E} \cap \bar{L}\right) \overline{\mathrm{W}}\left(\bar{U}(\Lambda) \cap \bar{G}_{E} \cap \bar{L}\right)$. Since $\bar{U}(\Lambda) \cap \bar{G}_{E} \cap \bar{L} \subset \bar{J}_{P}$, we are done.

Recall that we write $J_{\bar{L}}=i\left(J_{W}\right)$ and $\lambda_{\bar{L}}=i\left(\lambda_{W}\right)$. In order to prove that $\left(\bar{J}_{P}, \bar{\lambda}_{P}\right)$ is a cover of $\left(J_{\bar{L}}, \lambda_{\bar{L}}\right)$, the only thing remaining is to find a strongly $\left(\bar{P}, \bar{J}_{P}\right)$-positive element in the centre of $\bar{L}$ which supports an invertible element of the spherical Hecke algebra $\mathcal{H}\left(\bar{G}, \bar{\lambda}_{P}\right)$. To achieve this, we look at the $\bar{\lambda}_{P}$-spherical Hecke algebras of two parahoric subgroups whose intersections with $\bar{G}_{E}$ are non-conjugate maximal compact open subgroups of $\bar{G}_{E}$. Note that we could here have used instead the methods of [3] - indeed, the crucial ingredients (for example, the elements $w_{1}, w_{2}$ below) are the same.

Let $\mathcal{L}^{\prime(1)}$ be the self-dual $\mathfrak{o}_{E}$-lattice chain of $\mathfrak{o}_{F}$-period $e_{W}$ in $V^{\prime}=W \oplus W$ given by

$$
\cdots \supset L_{k}^{W} \oplus L_{k}^{W} \supset L_{k+1}^{W} \oplus L_{k+1}^{W} \supset \cdots,
$$

so that $\mathcal{L}^{\prime(1)}$ consists of every second lattice of $\mathcal{L}^{\prime}$. Let $\Lambda^{\prime(1)}$ be the self-dual $\mathfrak{o}_{E}$-lattice sequence in $V^{\prime}$ in which every lattice of $\mathcal{L}^{\prime(1)}$ occurs twice and with the indexing chosen such that

$$
\Lambda^{\prime(1)}(k)^{\#}=\Lambda^{\prime(1)}(1-k), \quad \text { for all } k \in \mathbb{Z} .
$$

Let $\Lambda^{(1)}$ be the $\mathfrak{o}_{F}$-lattice sequence in $V$ defined by

$$
\Lambda^{(1)}(k)=\Lambda^{\prime(1)}(k) \oplus \Lambda_{0}(2 k) .
$$

It is a self-dual lattice sequence of period $2 e_{W}=e / 2$ such that $\mathfrak{a}_{0}\left(\Lambda^{(1)}\right) \supset \mathfrak{a}_{0}(\Lambda)$. Put $\bar{K}_{1}=\bar{U}\left(\Lambda^{(1)}\right)$.

We define $\bar{K}_{2}=\bar{U}\left(\Lambda^{(2)}\right)$ by the same process, starting from the self-dual $\mathfrak{o}_{E}$-lattice chain $\mathcal{L}^{\prime(2)}$ in $V^{\prime}$ given by

$$
\cdots \supset L_{k}^{W} \oplus L_{k+1}^{W} \supset L_{k+1}^{W} \oplus L_{k+2}^{W} \supset \cdots
$$

Then $\bar{U}(\Lambda) \subset \bar{K}_{1} \cap \bar{K}_{2}$ so, in particular, $\bar{J}_{P} \subset \bar{K}_{1} \cap \bar{K}_{2}$.

Note that the element $w_{1}$ lies in $\overline{\mathrm{W}} \cap \bar{K}_{1}$. We also set $w_{2}=i\left(\varpi_{E}^{-1}, \mathbf{1}_{V_{0}}\right) w_{1} \in \overline{\mathrm{W}} \cap \bar{K}_{2}$.

Lemma 2.10. (i) $\mathcal{H}\left(\bar{K}_{1}, \bar{\lambda}_{P}\right)=\left\langle f_{1}, f_{w_{1}}\right\rangle$ where $f_{1}$ is supported on $\bar{J}_{P}$ and $f_{w_{1}}$ is supported on $\bar{J}_{P} w_{1} \bar{J}_{P}$.

(ii) $\mathcal{H}\left(\bar{K}_{2}, \bar{\lambda}_{P}\right)=\left\langle f_{1}, f_{w_{2}}\right\rangle$, with $f_{1}$ as in (i) and $f_{w_{2}}$ supported on $\bar{J}_{P} w_{2} \bar{J}_{P}$.

Proof Both parts follow from the following consideration. For $i=1,2$ the $\bar{K}_{i}$-intertwining of $\bar{\lambda}_{P}$ is given by

$$
I_{\bar{K}_{i}}\left(\bar{\lambda}_{P}\right)=\bar{J}_{P} \overline{\mathrm{W}} \bar{J}_{P} \cap \bar{K}_{i}=\bar{J}_{P}\left(\overline{\mathrm{W}} \cap \bar{K}_{i}\right) \bar{J}_{P} .
$$

But $\overline{\mathrm{W}} \cap \bar{K}_{i}=\left\{1, w_{i}\right\}$. Moreover, the restriction of $\bar{\lambda}_{P}$ to $\bar{J}_{P} \cap \bar{L}$ is irreducible and $w_{i}$ normalizes $\bar{J}_{P} \cap L$ so the intertwining space $I_{w_{i}}\left(\bar{\lambda}_{P}\right)$ is 1-dimensional. 
Lemma 2.11. Consider $f_{w_{1}}, f_{w_{2}}$ as elements of $\mathcal{H}\left(\bar{G}, \bar{\lambda}_{P}\right)$. Then the convolution $f_{w_{1}} * f_{w_{2}}$ is supported on $\bar{J}_{P} w_{1} w_{2} \bar{J}_{P}$.

Proof We know $f_{w_{1}} * f_{w_{2}}$ is supported on

$$
\begin{aligned}
& \bar{J}_{P} w_{1} \bar{J}_{P} w_{2} \bar{J}_{P} \\
& \quad=\bar{J}_{P}\left(w_{1}\left(\bar{J}_{P} \cap \bar{U}_{-}\right) w_{1}^{-1}\right)\left(w_{1}\left(\bar{J}_{P} \cap \bar{L}\right) w_{1}^{-1}\right) w_{1} w_{2}\left(w_{2}^{-1}\left(\bar{J}_{P} \cap \bar{U}\right) w_{2}\right) \bar{J}_{P} .
\end{aligned}
$$

From the explicit matrix descriptions in $1.4, \bar{J}_{P}$ contains $w_{1}\left(\bar{J}_{P} \cap \bar{U}_{-}\right) w_{1}^{-1}=w_{1}\left(\bar{H}^{1} \cap \bar{U}_{-}\right) w_{1}^{-1}$, $w_{1}\left(\bar{J}_{P} \cap \bar{L}\right) w_{1}^{-1}$ and $w_{2}^{-1}\left(\bar{J}_{P} \cap \bar{U}\right) w_{2}=w_{2}^{-1}\left(J^{1} \cap \bar{U}\right) w_{2}$, and the lemma follows.

We will prove that $f_{\zeta}:=f_{w_{1}} * f_{w_{2}}$ is invertible. To accomplish this we prove that $f_{w_{1}}$ and $f_{w_{2}}$ are each invertible. In each case we know

$$
f_{w_{i}} * f_{w_{i}}=c_{1 i} f_{1}+d_{i} f_{w_{i}}
$$

by Lemma 2.10. Thus, we only need to show $c_{1 i} \neq 0$ for each $i$.

Lemma 2.13. In equation $(2.12) c_{1 i} \neq 0$ for $i=1,2$.

Proof We treat only the case $i=1$, since the other case is identical. We need to check that $f_{w_{1}} * f_{w_{1}}(1) \neq 0$. Since

$$
f_{w_{1}}(x)= \begin{cases}0 & \text { if } x \notin \bar{J}_{P} w_{1} \bar{J}_{P} \\ \bar{\lambda}_{P}^{\vee}\left(j_{1}\right) f_{w_{1}}\left(w_{1}\right) \bar{\lambda}_{P}^{\vee}\left(j_{2}\right) & \text { if } x=j_{1} w_{1} j_{2}, \text { with } j_{1}, j_{2} \in \bar{J}_{P},\end{cases}
$$

we can write

$$
\begin{aligned}
f_{w_{1}} * f_{w_{1}}(1) & =\int_{\bar{K}_{1}} f_{w_{1}}(y) f_{w_{1}}\left(y^{-1}\right) d y \\
& =\frac{1}{\left|\bar{J}_{P} \cap w_{1} \bar{J}_{P}\right|} \int_{\bar{J}_{P} \times \bar{J}_{P}} f_{w_{1}}\left(j_{1} w_{1} j_{2}\right) f_{w_{1}}\left(j_{2}^{-1} w_{1}^{-1} j_{1}^{-1}\right) d j_{1} d j_{2} \\
& \left.=\frac{\left|\bar{J}_{P}\right|}{\left|\bar{J}_{P} \cap w_{1} \bar{J}_{P}\right|} \int_{\bar{J}_{P}} \bar{\lambda}_{P}^{\vee}\left(j_{1}\right) f_{w_{1}}\left(w_{1}\right) f_{w_{1}}\left(w_{1}^{-1}\right) \bar{\lambda}_{P}^{\vee}\left(j_{1}^{-1}\right)\right) d j_{1} .
\end{aligned}
$$

Now $w_{1}$ intertwines $\bar{\lambda}_{P}^{\vee}=\widetilde{i\left(\lambda_{W}^{\vee}\right)}$ and normalizes $\bar{J}_{P} \cap \bar{L}=i\left(J_{W}\right)$ so $f_{w_{1}}\left(w_{1}\right)$ is an equivalence $w_{1} i\left(\lambda_{W}^{\vee}\right) \simeq i\left(\lambda_{W}^{\vee}\right)$. But $w_{1}^{-1}=h_{1}^{-1} w_{1}$, where $h_{1}=i\left(\nu \sigma \varepsilon_{W}(\sigma), \mathbf{1}_{V_{0}}\right)$ and, by Proposition 1.1 $\nu \sigma \varepsilon_{W}(\sigma) \in J_{W}$; hence $w_{1}^{-1} \in J_{P} w_{1}$ and $f_{w_{1}}\left(w_{1}^{-1}\right)$ is an equivalence $i\left(\lambda_{W}^{\vee}\right) \simeq w_{1} i\left(\lambda_{W}^{\vee}\right)$. Thus $f_{w_{1}}\left(w_{1}\right) f_{w_{1}}\left(w_{1}^{-1}\right)$ is an equivalence of $i\left(\lambda_{W}^{\vee}\right)$ and hence a scalar $c \neq 0$. Thus

$$
f_{w_{1}} * f_{w_{1}}(1)=c \frac{\left|\bar{J}_{P}\right|^{2}}{\left|\bar{J}_{P} \cap w_{1} \bar{J}_{P}\right|} \neq 0
$$

Therefore, $f_{w_{1}}$ is invertible, as required.

Put $\zeta=i\left(\varpi_{E}, \mathbf{1}_{V_{0}}\right)$; since $\sigma \varepsilon_{W}(\sigma) \in J_{W}$ the function $f_{\zeta}$ is supported on $\bar{J}_{P} \zeta \bar{J}_{P}$.

Lemma 2.14. For each $k \in \boldsymbol{N}$, the $k$-fold convolution $f_{\zeta}^{k}$ is supported on $\bar{J}_{P} \zeta^{k} \bar{J}_{P}$. 
Proof This is by simple induction on $k$, since

$$
\begin{aligned}
& \bar{J}_{p} \zeta \bar{J}_{P} \zeta^{k} \bar{J}_{P} \\
& \quad=\bar{J}_{P}\left(\zeta\left(\bar{J}_{P} \cap \bar{U}\right) \zeta^{-1}\right)\left(\zeta\left(\bar{J}_{P} \cap \bar{L}\right) \zeta^{-1}\right) \zeta^{k+1}\left(\zeta^{-k}\left(\bar{J}_{P} \cap \bar{U}_{-}\right) \zeta^{k}\right) \bar{J}_{P},
\end{aligned}
$$

while $\bar{J}_{P}$ contains $\zeta\left(\bar{J}_{P} \cap \bar{U}\right) \zeta^{-1}, \zeta\left(\bar{J}_{P} \cap \bar{L}\right) \zeta^{-1}$ and $\zeta^{-k}\left(\bar{J}_{P} \cap \bar{U}_{-}\right) \zeta^{k}$ (cf. Lemma 2.11).

In particular, since $\varpi_{E}{ }^{e(E / F)}$ and $\varpi_{F}$ differ only by a unit of $E$, the function $f_{\zeta}^{e(E / F)}$ is an invertible element of $\mathcal{H}\left(\bar{G}, \bar{\lambda}_{P}\right)$ which is supported on the double coset $\bar{J}_{P} \zeta^{e(E / F)} \bar{J}_{P}=\bar{J}_{P} \zeta_{F} \bar{J}_{P}$, where

$$
\zeta_{F}=i\left(\varpi_{F} \mathbf{1}_{W}, \mathbf{1}_{V_{0}}\right)
$$

is a strongly $\left(\bar{P}, \bar{J}_{P}\right)$-positive element of the centre of $\bar{L}$. We conclude:

Theorem 2.15. Let $\pi_{W}$ be an irreducible supercuspidal representation of $G_{W} \cong G L_{M}(F)$, with $\pi_{W}^{\varepsilon_{W}} \simeq \pi_{W}$. Using the notation above, the pair $\left(\bar{J}_{P}, \bar{\lambda}_{P}\right)$ is a $\bar{G}$-cover of $\left(J_{\bar{L}}, \lambda_{\bar{L}}\right)$. In particular, it is an $\mathfrak{s}$-type, with $\mathfrak{s}=\left[\bar{L}, i\left(\pi_{W}\right)\right]_{\bar{G}}$.

Proof By construction, $\bar{J}_{P}$ is decomposed with respect to $\bar{P}$ so (i) and (ii) of [7, Definition 8.1] are satisfied for $\bar{P}$, and likewise for $\bar{P}_{-}$. By Lemma 2.13 the strongly $\left(\bar{P}, \bar{J}_{P}\right)$-positive element $\zeta_{F}$ supports an invertible element of $\mathcal{H}\left(\bar{G}, \bar{\lambda}_{P}\right)$. Thus $\left(\bar{J}_{P}, \bar{\lambda}_{P}\right)$ also satisfies (iii) of [7, Definition 8.1] for $\bar{P}$, and is therefore a $\bar{G}$-cover of $\left(J_{\bar{L}}, \lambda_{\bar{L}}\right)$. Then [7, Theorem 8.3] implies $\left(\bar{J}_{P}, \bar{\lambda}_{P}\right)$ is an $\mathfrak{s}$-type.

\section{$2.3 \quad$ Hecke Algebras}

In this section we derive results analogous to those of Chapter 5 of [6]. In particular, we show that the Hecke algebra of our type, $\mathcal{H}\left(\bar{G}, \bar{\lambda}_{P}\right)$ can be computed by using analogous computations in a case where the representation $\pi$ of $\bar{L}$ is of level zero. For many of these situations, [16] will give us the parameters of the Hecke algebra.

We fix $i=1$ or 2 and, in the notation of the previous section, we put $\Lambda^{\mathrm{M}}=\Lambda^{(i)}$. Then $\left[\Lambda^{\mathrm{M}}, n_{\mathrm{M}}, 0, \frac{1}{2} \beta\right]$ is a skew semisimple stratum and we set $\bar{J}_{\mathrm{M}}=\bar{J}\left(\frac{1}{2} \beta, \Lambda^{\mathrm{M}}\right)$, and similarly $\bar{J}_{\mathrm{M}}^{1}$ and $\bar{H}_{\mathrm{M}}^{1}$. Let $\bar{\theta}_{\mathrm{M}}$ denote the transfer of $\bar{\theta}$ to $\bar{H}_{\mathrm{M}}^{1}$. By [22, Corollary 4.2], there is a unique irreducible representation $\bar{\eta}_{\mathrm{M}}$ of $\bar{J}_{M}^{1}$ which extends $\bar{\theta}_{\mathrm{M}}$, and by [25, Theorem 4.1], we may choose a $\beta$-extension, $\bar{\kappa}_{\mathrm{M}}$ of $\bar{\eta}_{\mathrm{M}}$ to $\bar{J}_{\mathrm{M}}-$ we recall here what we mean by $\beta$-extension:

Recall the lattice sequence $\Lambda^{\mathrm{m}}$ from $₫ 1.4$ such that $\bar{U}\left(\Lambda^{\mathrm{m}}\right) \cap \bar{G}_{E}$ is an Iwahori subgroup of $\bar{U}(\Lambda) \cap \bar{G}_{E}$; then we have $\bar{\theta}^{\mathrm{m}}$ the transfer of $\bar{\theta}$ and $\bar{\eta}^{\mathrm{m}}$ the unique irreducible representation of $\bar{J}^{1}\left(\frac{1}{2} \beta, \Lambda^{\mathrm{m}}\right)$ containing $\bar{\theta}^{\mathrm{m}}$. We abbreviate $\bar{U}(\Lambda) \cap \bar{G}_{E}=\bar{U}\left(\Lambda_{\mathfrak{o}_{E}}\right)$ (and similarly for other lattice sequences) and define $\hat{J}_{M}=\bar{U}\left(\Lambda_{\mathfrak{o}_{E}}\right) \bar{J}_{M}^{1}$ and $\hat{J}_{M}^{1}=\bar{U}^{1}\left(\Lambda_{\mathfrak{o}_{E}}^{\mathrm{m}}\right) \bar{J}_{\mathrm{M}}^{1}$. Thus, $\bar{J}_{\mathrm{M}} \supset \hat{J}_{\mathrm{M}} \supset \hat{J}_{M}^{1} \supset \bar{J}_{M}^{1}$ and $\hat{J}_{M}^{1}$ is a pro- $p$ Sylow subgroup of $\bar{J}_{\mathrm{M}}$. By [25, Proposition 3.7], there is a unique irreducible representation, $\hat{\eta}_{M}$ of $\hat{J}_{\mathrm{M}}^{1}$ which extends $\bar{\eta}_{\mathrm{M}}$ and such that $\hat{\eta}_{\mathrm{M}}$ and $\bar{\eta}^{\mathrm{m}}$ induce equivalent irreducible representations of $\bar{U}^{1}\left(\Lambda_{\mathrm{m}}\right)$. Then a $\beta$-extension $\bar{\kappa}_{\mathrm{M}}$ is an extension to $\bar{J}_{\mathrm{M}}$ of $\hat{\eta}_{\mathrm{M}}$.

Similarly (as in [6, Proposition 5.2.5] - see [25, Lemma 4.2]), there is a unique irreducible representation, $\hat{\mu}_{\mathrm{M}}$ of $\hat{J}_{\mathrm{M}}$ which extends $\bar{\eta}_{\mathrm{M}}$ and such that

$$
\operatorname{Ind}_{\hat{J}_{\mathrm{M}}}^{\bar{U}\left(\Lambda_{\mathfrak{o}_{E}}\right) \bar{U}^{1}(\Lambda)} \hat{\mu}_{\mathrm{M}} \simeq \operatorname{Ind}_{\bar{J}_{P}}^{\bar{U}\left(\Lambda_{\mathfrak{o}_{E}}\right) \bar{U}^{1}(\Lambda)} \bar{\kappa}_{P} .
$$

Moreover, as in [6, Proposition 5.2.6], we have $\left.\hat{\mu}_{\mathrm{M}}\right|_{\hat{J}_{\mathrm{M}}^{1}}=\hat{\eta}_{\mathrm{M}}$. The following Lemma will be crucial: 
Lemma 2.17. Let $\bar{\tau}_{1}, \bar{\tau}_{2}$ be irreducible representations of $\bar{U}\left(\Lambda_{\mathfrak{o}_{E}}\right) / \bar{U}^{1}\left(\Lambda_{\mathfrak{o}_{E}}\right)$, which we regard as representations of $\bar{J}_{P}$ or $\hat{J}_{\mathrm{M}}$. Then there is a Hecke algebra isomorphism

$$
\begin{aligned}
\mathcal{H}\left(\bar{G}, \bar{\kappa}_{P} \otimes \bar{\tau}_{1}, \bar{\kappa}_{P} \otimes \bar{\tau}_{2}\right) & \longrightarrow \mathcal{H}\left(\bar{G}, \hat{\mu}_{\mathrm{M}} \otimes \bar{\tau}_{1}, \hat{\mu}_{\mathrm{M}} \otimes \bar{\tau}_{2}\right) \\
\bar{f} & \longmapsto \hat{f},
\end{aligned}
$$

which preserves support: if $\bar{f}$ has support $\bar{J}_{P} g \bar{J}_{P}$, for some $g \in \bar{G}_{E}$, then $\hat{f}$ has support $\hat{J}_{\mathrm{M}} g \hat{J}_{\mathrm{M}}$.

Proof Note that we can also think of $\bar{\tau}_{j}$ as a representation of $\bar{U}\left(\Lambda_{\mathfrak{o}_{E}}\right) \bar{U}^{1}(\Lambda)$, and we get

$$
\operatorname{Ind}_{\bar{J}_{P}}^{\bar{U}\left(\Lambda_{\mathfrak{o}_{E}}\right) \bar{U}^{1}(\Lambda)}\left(\bar{\kappa}_{P} \otimes \bar{\tau}_{j}\right)=\left(\operatorname{Ind}_{\bar{J}_{P}}^{\bar{U}\left(\Lambda_{\mathfrak{o}_{E}}\right) \bar{U}^{1}(\Lambda)} \bar{\kappa}_{P}\right) \otimes \bar{\tau}_{j}
$$

and similarly for $\hat{\mu}_{\mathrm{M}}$. The first assertion is then immediate from (2.16), by Lemma A.1 in the Appendix. The assertion concerning supports is a mild generalization of, for example, [25, Lemma 6.1 and Proposition 7.1] (see also [6, Proposition 5.5.13]), using Lemma A.1 in place of [6, Corollary 4.1.5]. More precisely, put $\bar{\kappa}=\operatorname{Ind} \bar{J}_{P} \bar{\kappa}_{P}$ (which is irreducible, since $I_{\bar{G}}\left(\bar{\kappa}_{P}\right) \subset$ $\left.I_{\bar{G}}\left(\bar{\eta}_{P}\right)=\bar{J}_{P} \bar{G}_{E} \bar{J}_{P}\right)$, and consider $\bar{\tau}_{j}$ also as representations of $\bar{J}_{\mathrm{M}}$; then the results cited are analogous to showing that there are support-preserving isomorphisms from each Hecke algebra to $\mathcal{H}\left(\bar{G}, \bar{\kappa} \otimes \bar{\tau}_{1}, \bar{\kappa} \otimes \bar{\tau}_{2}\right)$, and our isomorphism is the composition of these.

Using this, we obtain:

Lemma 2.18. There is a choice of $\kappa_{W}$ for which $\kappa_{W} \circ \widetilde{\sigma} \simeq \kappa_{W}$.

Proof Recall that we have fixed $i=1$ or 2 ; in the notation of the previous section, we put $w=w_{i}$. Note that, by Proposition 2.8, $w$ intertwines the representation $\bar{\eta}_{P}=\widetilde{i\left(\eta_{W}\right)}$ of $\bar{J}_{P}$.

We fix some choice of $\kappa_{W}$. Now $\kappa_{W} \circ \widetilde{\sigma}$ is also a $\beta$-extension of $\eta_{W}$ so $\kappa_{W} \circ \widetilde{\sigma} \simeq \kappa_{W} \otimes \chi_{W}$, for some character $\chi_{W}$ of $U\left(\mathfrak{B}_{W}\right) / U^{1}\left(\mathfrak{B}_{W}\right) \cong J_{W} / J_{W}^{1}$ which factors through the determinant $\operatorname{det}_{B_{W} / E}$. Then

$$
{ }^{w} i\left(\kappa_{W}\right) \simeq i\left(\kappa_{W} \circ \widetilde{\sigma}\right)=i\left(\kappa_{W}\right) \otimes i\left(\chi_{W}\right)
$$

and $w$ intertwines $\bar{\kappa}_{P}$ with $\bar{\kappa}_{P} \otimes \bar{\chi}_{P}\left(\right.$ where $\bar{\chi}_{P}=\widetilde{i\left(\chi_{W}\right)}$ on $\left.\bar{J}_{P}\right)$, since both are trivial on the unipotent parts of $\bar{J}_{P}$. We also put $\hat{\chi}_{\mathrm{M}}=\widetilde{i\left(\chi_{W}\right)}$ on $\hat{J}_{\mathrm{M}}$. Then Lemma 2.17 (applied with $\bar{\tau}_{1}$ the trivial representation and $\left.\bar{\tau}_{2}=\widetilde{i\left(\chi_{W}\right)}\right)$ implies that $w$ intertwines $\hat{\mu}_{\mathrm{M}}$ with $\hat{\mu}_{\mathrm{M}} \otimes \hat{\chi}_{\mathrm{M}}$.

Recall that we have $\left.\bar{\kappa}_{M}\right|_{\hat{J}_{M}^{1}}=\hat{\eta}_{M}=\left.\hat{\mu}_{M}\right|_{\hat{J}_{M}^{1}}$, by construction of $\beta$-extensions in [25, Theorem 4.1]. Thus, if we let $\hat{\kappa}_{M}=\left.\bar{\kappa}_{M}\right|_{\hat{J}_{M}}$, we must have $\hat{\kappa}_{M} \simeq \hat{\mu}_{M} \otimes \bar{\psi}_{M}$ for a character $\bar{\psi}_{M}$ of $\bar{U}\left(\Lambda_{\mathfrak{o}_{E}}\right) / \bar{U}^{1}\left(\Lambda_{\mathfrak{o}_{E}}^{\mathrm{m}}\right)$, that is, a character of the Siegel Levi subgroup of $\bar{U}\left(\Lambda_{\mathfrak{o}_{E}}^{\mathrm{M}}\right) / \bar{U}^{1}\left(\Lambda_{\mathfrak{o}_{E}}^{\mathrm{M}}\right)$ which is trivial on the maximal unipotent subgroup $\bar{U}^{1}\left(\Lambda_{\mathfrak{o}_{E}}^{\mathrm{m}}\right) / \bar{U}^{1}\left(\Lambda_{\mathfrak{o}_{E}}^{\mathrm{M}}\right)$. Then $\bar{\psi}_{\mathrm{M}}$ factorizes through the determinant on the Levi subgroup and we can write $\bar{\psi}_{\mathrm{M}}=\widehat{i\left(\psi_{W}\right)}$, for some character $\psi_{W}$ of $J_{W} / J_{W}^{1}$.

Now $w \in \bar{J}_{M}$ so $w$ certainly intertwines $\hat{\kappa}_{\mathbf{M}}$ with itself. Hence $w$ intertwines $\hat{\mu}_{\mathbf{M}} \otimes \bar{\psi}_{\mathrm{M}}$ with $\hat{\mu}_{M} \otimes \hat{\chi}_{M} \otimes{ }^{w} \bar{\psi}_{M}$. Chasing back through the isomorphism of Lemma 2.17, we see that $w$ intertwines $\bar{\kappa}_{P} \otimes \bar{\psi}_{\mathrm{M}}$ with $\bar{\kappa}_{P} \otimes \hat{\chi}_{\mathrm{M}} \otimes{ }^{w} \bar{\psi}_{\mathrm{M}}$. Since $w$ normalizes $\bar{L}$, this implies that conjugation by $w$ gives an equivalence $\kappa_{W} \otimes \psi_{W} \simeq \kappa_{W} \otimes \chi_{W}\left(\psi_{W} \circ \widetilde{\sigma}\right)$. By [6, Theorem 5.2.2], we deduce that $\psi_{W}=\chi_{W}\left(\psi_{W} \circ \widetilde{\sigma}\right)$ and, in particular, $\kappa_{W} \otimes \psi_{W}$ is a $\beta$-extension with the required property. 
We now choose $\kappa_{W}$ as in Lemma 2.18 and make the same constructions as before: $\bar{\kappa}_{P}, \bar{\kappa}$ and $\hat{\mu}_{\mathrm{M}}$. Comparing $\hat{\mu}_{\mathrm{M}}$ with $\hat{\kappa}_{\mathrm{M}}$, we again get a character $\bar{\psi}_{\mathrm{M}}$ but now with the property that ${ }^{w} \bar{\psi}_{\mathrm{M}}=\bar{\psi}_{\mathrm{M}}$. If $E / E_{0}$ is unramified then this implies that $\bar{\psi}_{\mathrm{M}}={ }^{w} \bar{\psi}_{0} \bar{\psi}_{0}$, for some character $\bar{\psi}_{0}$. Then $\bar{\psi}_{0}$ extends to a character of $J_{\mathrm{M}}$ and, replacing $\bar{\kappa}_{\mathrm{M}}$ by $\bar{\kappa}_{\mathrm{M}} \otimes \bar{\psi}_{0}^{-1}$, we may assume $\bar{\psi}_{\mathrm{M}}=1$. In the ramified case, the condition on $\bar{\psi}_{\mathrm{M}}$ is that $\bar{\psi}_{\mathrm{M}}^{2}=1$ but it is (at least in principle) possible that $\bar{\psi}_{\mathrm{M}} \neq 1$.

We write $\bar{\psi}_{\mathrm{M}}=\widetilde{i\left(\psi_{W}\right)}$; then we may assume $\psi_{W}^{2}=1$.

Proposition 2.19. With the notation as above, there is an algebra isomorphism

$$
\mathcal{H}\left(\bar{J}_{\mathrm{M}}, \bar{\lambda}_{P}\right) \stackrel{\sim}{\stackrel{\sim}{\longrightarrow}} \mathcal{H}\left(\bar{U}\left(\Lambda_{\mathfrak{o}_{E}}^{\mathrm{M}}\right) / \bar{U}^{1}\left(\Lambda_{\mathfrak{o}_{E}}^{\mathrm{M}}\right), i\left(\widetilde{\rho_{W} \otimes \psi_{W}}\right)\right)
$$

which preserves support: if $\bar{f} \in \mathcal{H}\left(\bar{J}_{\mathrm{M}}, \bar{\lambda}_{P}\right)$ has support $\bar{J}_{P} b \bar{J}_{P}$, for some $b \in \bar{J}_{\mathrm{M}} \cap \bar{G}_{E}=\bar{U}\left(\Lambda_{\mathfrak{o}_{E}}^{\mathrm{M}}\right)$, then $\iota(\bar{f})$ has support $\left(\bar{U}\left(\Lambda_{\mathfrak{o}_{E}}\right) / \bar{U}^{1}\left(\Lambda_{\mathfrak{o}_{E}}^{\mathrm{M}}\right)\right) b\left(\bar{U}\left(\Lambda_{\mathfrak{o}_{E}}\right) / \bar{U}^{1}\left(\Lambda_{\mathfrak{o}_{E}}^{\mathrm{M}}\right)\right)$.

Proof From Lemma 2.17, we get a support-preserving isomorphism

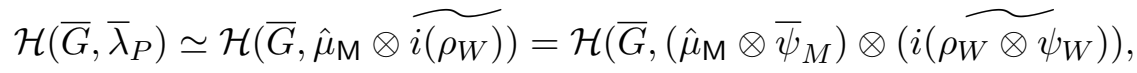

where we are using the fact that $\psi_{W}^{2}=1$. On the other hand, we have support-preserving isomorphisms

$$
\begin{aligned}
\mathcal{H}\left(\bar{J}_{\mathrm{M}},\left(\hat{\mu}_{\mathrm{M}} \otimes \bar{\psi}_{M}\right) \otimes\left(i\left(\widetilde{\rho_{W} \otimes \psi_{W}}\right)\right)\right. & \simeq \mathcal{H}\left(\bar{J}_{\mathrm{M}}, i\left(\widetilde{\rho_{W} \otimes \psi_{W}}\right)\right) \\
& \simeq \mathcal{H}\left(\bar{U}\left(\Lambda_{\mathfrak{o}_{E}}^{\mathrm{M}}\right) / \bar{U}^{1}\left(\Lambda_{\mathfrak{o}_{E}}^{\mathrm{M}}\right), i\left(\widetilde{\rho_{W} \otimes \psi_{W}}\right)\right),
\end{aligned}
$$

where the first isomorphism follows from the fact that $\hat{\mu}_{M} \otimes \bar{\psi}_{M}=\hat{\kappa}_{M}$ extends to a representation $\bar{\kappa}_{\mathrm{M}}$ of $\bar{J}_{\mathrm{M}}$ (cf. [6, Lemma 5.6.3]), and the second by reduction modulo $\bar{J}_{\mathrm{M}}^{1}$, since $\bar{J}_{\mathrm{M}} / \bar{J}_{\mathrm{M}}^{1} \cong$ $\bar{U}\left(\Lambda_{\mathfrak{o}_{E}}^{\mathrm{M}}\right) / \bar{U}^{1}\left(\Lambda_{\mathfrak{o}_{E}}^{\mathrm{M}}\right)$. Putting these isomorphisms together gives the isomorphism of the Proposition.

Remarks 2.20. (i) Note that, writing $\mathfrak{B}_{M}^{\prime}$ for the self-dual $\mathfrak{o}_{E}$-order $\mathfrak{a}_{0}\left(\Lambda^{\mathrm{M}}\right) \cap B^{\prime}$, we have

$$
\begin{aligned}
\bar{U}\left(\Lambda_{\mathfrak{o}_{E}}^{\mathrm{M}}\right) / \bar{U}^{1}\left(\Lambda_{\mathfrak{o}_{E}}^{\mathrm{M}}\right) & \cong \bar{U}\left(\mathfrak{B}_{\mathrm{M}}^{\prime}\right) / \bar{U}^{1}\left(\mathfrak{B}_{\mathrm{M}}^{\prime}\right) \times \bar{G}_{0} / \bar{G}_{0}^{1} \text { and } \\
\bar{U}\left(\Lambda_{\mathfrak{o}_{E}}\right) / \bar{U}^{1}\left(\Lambda_{\mathfrak{o}_{E}}^{\mathrm{M}}\right) & \cong \bar{U}\left(\mathfrak{B}^{\prime}\right) / \bar{U}^{1}\left(\mathfrak{B}_{\mathrm{M}}^{\prime}\right) \times \bar{G}_{0} / \bar{G}_{0}^{1},
\end{aligned}
$$

where $\bar{G}_{0}^{1}$ is the pro- $p$ radical of the anisotropic group $\bar{G}_{0}$. Then we have an isomorphism

$$
\mathcal{H}\left(\bar{U}\left(\Lambda_{\mathfrak{o}_{E}}^{\mathrm{M}}\right) / \bar{U}^{1}\left(\Lambda_{\mathfrak{o}_{E}}^{\mathrm{M}}\right), i\left(\widetilde{\rho_{W} \otimes \psi_{W}}\right)\right) \simeq \mathcal{H}\left(\bar{U}\left(\mathfrak{B}_{\mathrm{M}}^{\prime}\right) / \bar{U}^{1}\left(\mathfrak{B}_{\mathrm{M}}^{\prime}\right), i\left(\widetilde{\rho_{W} \otimes \psi_{W}}\right)\right) .
$$

The quotient $\bar{U}\left(\mathfrak{B}_{\mathrm{M}}^{\prime}\right) / \bar{U}^{1}\left(\mathfrak{B}_{\mathrm{M}}^{\prime}\right)$ is a unitary (if $E / E_{0}$ is unramified), symplectic or orthogonal group over $k_{E_{0}}$ and the Hecke algebra on the right is described in [13. Alternatively, reduction modulo $\bar{U}^{1}\left(\mathfrak{B}_{\mathrm{M}}^{\prime}\right)$ gives a support-preserving isomorphism

$$
\mathcal{H}\left(\bar{U}\left(\mathfrak{B}_{\mathrm{M}}^{\prime}\right) / \bar{U}^{1}\left(\mathfrak{B}_{\mathrm{M}}^{\prime}\right), i\left(\widetilde{\rho_{W} \otimes \psi_{W}}\right)\right) \simeq \mathcal{H}\left(\bar{U}\left(\mathfrak{B}_{\mathrm{M}}^{\prime}\right), i\left(\widetilde{\rho_{W} \otimes \psi_{W}}\right)\right),
$$

and the latter is described in [18]. This will allow us, in a future work, to compute explicitly the parameters for the Hecke algebra. 
(ii) Since (in the case where $E / E_{0}$ is ramified) we have $\psi_{W}^{2}=1$, we may replace our choice of $\kappa_{W}$ by $\kappa_{W} \otimes \psi_{W}$ (which has the same property of being fixed by $\tilde{\sigma}$ ); this replaces $\rho_{W}$ by $\rho_{W} \otimes \psi_{W}$, another self-dual cuspidal representation of $U\left(\mathfrak{B}_{W}\right) / U^{1}\left(\mathfrak{B}_{W}\right)$, and we lose the character $\psi_{W}$ from the RHS of the isomorphism in Proposition 2.19. However, we cannot do this independently for the two choices $\Lambda^{(1)}, \Lambda^{(2)}$ for $\Lambda^{\mathrm{M}}$. In particular, if we choose (as we always can) to dispose with the character $\psi_{W}$ in one case, then it may a priori still be non-trivial in the other.

(iii) Since $\left(\bar{J}_{P}, \bar{\lambda}_{P}\right)$ is a cover of $\left(J_{\bar{L}}, \lambda_{\bar{L}}\right)$, by [7, Corollary 7.12] we have a canonical embedding of Hecke algebras $t_{P}: \mathcal{H}\left(\bar{L}, \lambda_{\bar{L}}\right) \hookrightarrow \mathcal{H}\left(\bar{G}, \bar{\lambda}_{P}\right)$ and we identify $\mathcal{H}\left(\bar{L}, \lambda_{\bar{L}}\right)$ (which is just the algebra of Laurent polynomials in a single variable) with its image $\mathcal{B}_{P}$. We also put $\mathcal{K}=\mathcal{H}\left(\bar{J}_{\mathrm{M}}, \bar{\lambda}_{P}\right)$. Then [9, Theorem 1.5] implies that the map

$$
\begin{aligned}
\mathcal{B}_{P} \otimes_{\mathbb{C}} \mathcal{K} & \rightarrow \mathcal{H}\left(\bar{G}, \bar{\lambda}_{P}\right) \\
f \otimes \phi & \mapsto f * \phi
\end{aligned}
$$

is an isomorphism of $\left(\mathcal{B}_{P}, \mathcal{K}\right)$-bimodules.

We conclude with a description of the Hecke algebra as a convolution algebra. We put $\mathbf{S}=\left\{w_{1}, w_{2}\right\}$ and recall that we have elements $f_{w_{i}} \in \mathcal{H}\left(\bar{G}, \bar{\lambda}_{P}\right)$ with support $\bar{J}_{P} w_{i} \bar{J}_{P}$.

Theorem 2.21 (cf. [1, (1.11) Théorème]). The algebra $\mathcal{H}\left(\bar{G}, \bar{\lambda}_{P}\right)$ is a convolution algebra on $(\overline{\mathrm{W}}, \mathrm{S})$. As a vector space, it has basis $f_{w}=f_{s_{1}} * \cdots * f_{s_{r}}$, where $w=s_{1} \cdots s_{r}$ is a reduced decomposition of $w$ in $\overline{\mathrm{W}}$. It is generated as an algebra by $f_{w_{1}}$ and $f_{w_{2}}$, with relations

$$
f_{w_{i}} * f_{w_{i}}=c_{1 i} f_{w_{i}}+d_{i} f_{w_{i}}, \quad \text { for } i=1,2 .
$$

Proof The proof is the same as that of [1, (1.11) Théorème].

\section{A Intertwining and Hecke algebras}

In this short appendix we give the statement of a result, which is a mild generalization of [6, Proposition 4.1.3, Corollary 4.1.5]. The notation here is independent of that in the rest of this paper.

Let $G$ be a unimodular locally compact totally disconnected topological group with a countable base of open sets, and fix a Haar measure on $G$. Let $K$ be a compact open subgroup of $G$ and $\left(\rho_{i}, W_{i}\right)$ a continuous finite-dimensional representation of $K$, for $i=1,2$.

Let $\mathcal{H}\left(G, \rho_{1}, \rho_{2}\right)$ be the complex vector space of compactly supported functions $\phi: G \rightarrow \operatorname{Hom}_{\mathbb{C}}\left(W_{2}^{\vee}, W_{1}^{\vee}\right)$ satisfying

$$
\phi\left(k_{1} g k_{2}\right)=\rho_{1}^{\vee}\left(k_{1}\right) \circ \phi(g) \circ \rho_{2}^{\vee}\left(k_{2}\right), \quad \text { for all } k_{1}, k_{2} \in K, g \in G .
$$

Then $\mathcal{H}\left(G, \rho_{1}, \rho_{2}\right)$ is an $\left(\mathcal{H}\left(G, \rho_{1}\right), \mathcal{H}\left(G, \rho_{2}\right)\right)$-bimodule, under convolution of functions. If $g \in G$ then we write $\mathcal{H}\left(G, \rho_{1}, \rho_{2}\right)_{g}$ for the subspace of functions which are null outside $K g K$.

Recall that the $G$-intertwining of $\rho_{1}$ with $\rho_{2}$ is given by

$$
I_{G}\left(\rho_{1}, \rho_{2}\right)=\left\{g \in G: \operatorname{Hom}_{K \cap^{g} K}\left(\rho^{g}, \rho_{2}\right) \neq 0\right\} .
$$

The result we need is the following: 
Lemma A.1 (cf. [6, 4.1.1-5]). In the above situation, let $g \in G$.

(i) There is a function $\phi \in \mathcal{H}\left(G, \rho_{1}, \rho_{2}\right)$ with $\phi(g) \neq 0$ if and only if $g^{-1} \in I_{G}\left(\rho_{1}, \rho_{2}\right)$. Moreover, if $g$ satisfies these conditions then there is a canonical isomorphism of vector spaces between $\operatorname{Hom}_{K \cap K^{g}}\left(W_{1}, W_{2}^{g}\right)$ and $\mathcal{H}\left(G, \rho_{1}, \rho_{2}\right)_{g}$.

(ii) Let $H$ be a compact open subgroup containing $K$. Then there is a canonical isomorphism

$$
\mathcal{H}\left(G, \rho_{1}, \rho_{2}\right) \simeq \mathcal{H}\left(G, \operatorname{Ind}_{K}^{H} \rho_{1}, \operatorname{Ind}_{K}^{H} \rho_{2}\right)
$$

of $\left(\mathcal{H}\left(G, \rho_{1}\right), \mathcal{H}\left(G, \rho_{2}\right)\right)$-bimodules. This restricts to an isomorphism

$$
\mathcal{H}\left(G, \operatorname{Ind}_{K}^{H} \rho_{1}, \operatorname{Ind}_{K}^{H} \rho_{2}\right)_{g} \simeq \coprod_{\substack{g^{\prime} \in K \backslash G / K \\ H g^{\prime} H=H g H}} \mathcal{H}\left(G, \rho_{1}, \rho_{2}\right)_{g^{\prime}} .
$$

The proof is very similar to those of [6, 4.1.1-5]; one point to note is that we are using the canonical isomorphisms of [6, Proposition 4.1.3] to identify $\mathcal{H}\left(G, \rho_{i}\right)$ with $\mathcal{H}\left(G, \operatorname{Ind}_{K}^{H} \rho_{i}\right)$, for $i=1,2$, so that $\mathcal{H}\left(G, \operatorname{Ind}_{K}^{H} \rho_{1}, \operatorname{Ind}_{K}^{H} \rho_{2}\right)$ is indeed an $\left(\mathcal{H}\left(G, \rho_{1}\right), \mathcal{H}\left(G, \rho_{2}\right)\right)$-bimodule.

\section{References}

[1] Blasco L., Blondel C., Types induits des paraboliques maximaux de $S p_{4}(F)$ et $G S p_{4}(F)$, Ann. Inst. Fourier (Grenoble) 49(6) (1999) 1805-1851.

[2] Blondel C., Critère d'injectivité pour l'application de Jacquet, C. R. Acad. Sci. Paris Sér. I Math. 325 (1997) 1149-1152.

[3] Blondel C., $S p(2 N)$-covers for self-contragredient supercuspidal representations of $G L(N)$, Ann. Sci. École Norm. Sup. (4) 37(4) (2004) 533-558.

[4] Borel A., Admissible representations of a semi-simple group over a local field with vectors fixed under an Iwahori subgroup, Invent. Math. 35 (1976) 233-259.

[5] Bushnell C.J., Henniart G., Local tame lifting for $G L(N)$ I: simple characters, Publ. Math. IHES 83 (1996) 105-233.

[6] Bushnell C.J., Kutzko P.C., The admissible dual of $G L(N)$ via compact open subgroups, Princeton University Press, 1993.

[7] Bushnell C.J., Kutzko P.C., Smooth representations of reductive p-adic groups: structure theory via types, Proc. London Math. Soc. (3) 77 (1998) 582-634.

[8] Bushnell C.J., Kutzko P.C., Semisimple types, Compositio Math. 119 (1999) 53-97.

[9] Bushnell C.J., Kutzko P.C., Types in reductive $p$-adic groups: the Hecke algebra of a cover, Proc. Amer. Math. Soc. 129(2) (2000) 601-607.

[10] Glauberman G., Correspondences of characters for relatively prime operator groups, Canad. J. Math. (4) 20 (1968) 1465-1488. 
[11] GeL'Fand I.M., Kazhdan D.A.,Representations of the group $G L(n, K)$ where $K$ is a local field, in Lie groups and their representations (Proc. Summer School on Group Representations of the Bolya: János Math. Soc., Budapest, 1971), 95-118, Halsted, New York, 1975.

[12] Harris M., TAYlor R., The geometry and cohomology of some simple Shimura varieties, Ann. Math. Studies, Princeton University Press, 2001.

[13] Howlett R.B., Lehrer G.I., Induced cuspidal representations and generalised Hecke rings, Invent. Math. 58(1) (1980) 37-64.

[14] Henniart G., Une preuve simple des conjectures de Langlands pour $\mathrm{GL}_{n}$ sur un corps $p$-adique, Invent. Math. 139(2) (2000) 439-455.

[15] Henniart G., Correspondance de Langlands et fonctions $L$ des carrés extérieur et symétrique, Prépublication M/03/20 de l'IHES March 2003, www.ihes.fr/PREPRINTS/M03/M03-20.pdf

[16] Kutzko P.C, Morris L., Parabolic induction and Hecke algebras, to appear.

[17] Morris L., Tamely ramified supercuspidal representations of classical groups I: Filtrations, Ann. Sci. École Norm. Sup. (4) 24 (1991) 705-738.

[18] Morris L., Tamely ramified intertwining algebras, Invent. Math. 114 (1993) 1-54.

[19] Roche A., Types and Hecke algebras for principal series representations of split reductive p-adic groups, Ann. Sci. École Norm. Sup. (4) 31(3) (1998), 361-413.

[20] Sécherre V., Représentations lisses de GL $(m, D)$, II: $\beta$-extensions, Compositio Math. 141 (2005) 1531-1550.

[21] Shahidi F., Twisted Endoscopy and reducibility of induced representations for $p$-adic groups, Duke Math.J 66 (1992) 1-41.

[22] Stevens S., Intertwining and supercuspidal types for classical p-adic groups, Proc. London Math. Soc. (3) 83 (2001) 120-140.

[23] Stevens S., Double coset decompositions and intertwining, Manuscripta Math. 106 (2001) 349-364.

[24] Stevens S., Semisimple characters for p-adic classical groups, Duke Math. J., 127(1) (2005) $123-173$.

[25] Stevens S., The supercuspidal representations of $p$-adic classical groups, Preprint, July 2006, arXiv:math.RT/0607622

David Goldberg,

Department of Mathematics,

Purdue University,

West Lafayette,

IN 47907-1395,

USA

goldberg@math.purdue.edu
Philip Kutzko,

Department of Mathematics, University of Iowa,

Iowa City,

IA 52242-1419, USA

philip-kutzko@uiowa.edu
Shaun Stevens,

School of Mathematics, University of East Anglia, Norwich,

NR4 7TJ,

United Kingdom

Shaun.Stevens@uea.ac.uk 